\title{
Complication from Desensitization
}

\author{
Duck Jong Han
}

Q: What kinds of complication can be occurred from various desensitization medication or method?

1. Medical complication

- Complication of rituximab (antiCD20 mAb)

- Complication of bortezomib

- Complication of IVIg

- Complication of eculizumab

- Complication of $\mathrm{C} 1$ esterase inhibitor

- Complication of IgG endopeptidase

- Complication of anti-IL-6R mAb

- Complication of plasmapheresis

2. Surgical complication

\section{Complication from Rituximab}

\section{Q: 1. What kind of side effect doses the rituximab have?}

2. Regarding the infection following the rituximab, is it related with the dose of rituximab?

\section{J. Han $(\bowtie)$}

Department of General Surgery, Asan Medical Center, Seoul, South Korea

e-mail: djhan@amc.seoul.kr
Rituximab is a mouse-human chimeric antibody that targets CD20, and recognized as a useful drug for the treatment of B cell non-Hodgkin's lymphoma and its use has been extended to such diseases as idiopathic thrombocytopenic purpura, thrombotic thrombocytopenic purpura, chronic rheumatoid arthritis, and ANCA-associated vasculitides. Recently, B cell depletion with rituximab has been an important component of desensitization for $\mathrm{ABO}$ incompatible or positive crossmatch kidney transplantation [1]. However rituximab has diverse side effects at immediate after use or even up to a year.

\section{Infection}

Common side effects of rituximab are infusionrelated reactions and infections. Rituximab has been associated with serious infections, including Pneumocystis jirovecii pneumonia (PJP) and the reactivation of hepatitis $\mathrm{B}$ virus (HBV) and tuberculosis (TB). The risk of infection appears to be the result of a variety of mechanisms, including prolonged $\mathrm{B}$ cell depletion, B-cell-Tcell crosstalk, panhypogammaglobulinemia, lateonset neutropenia, and blunting of the immune response after vaccination. Particular attention should be given to strategies to minimize the risk of infectious complications, including vaccinating 
against bacterial and viral pathogens, monitoring white cell count and immunoglobulin levels, prophylaxis against PJP, and screening for HBV and TB [2].

Chung et al. [3] reported that the overall prevalence of infection was significantly higher, and the infection-free survival rate was lower, in the group of combined rituximab, plasmapheresis, and IVIg (RPI), compared with the rituximab alone or control groups $(P<0.05)$. A trend toward more severe bacterial infections was seen in the RPI group compared with the other groups, and fungal infections developed only in the RPI group.

Therefore the use of combined RTX and PP therapy pretransplant significantly increases the risk for posttransplant infection.

Regarding the rituximab dose effect on infection, Nakao [4] reported in $\mathrm{ABO}$ mismatched patients that an initial rituximab dosage of $100 \mathrm{mg} /$ body (for titers below 64) or $200 \mathrm{mg} /$ body (for titers above 128) was administered 2 weeks before transplantation. If the percentage of peripheral B lymphocytes remained greater than $0.5 \%$, additional rituximab (100 or $200 \mathrm{mg}$ ) was administered. Nine patients received rituximab $100 \mathrm{mg} /$ body (low-dose rituximab [LDR] group). Overall survival and graft survival rates did not differ significantly between the LDR group and other cases. The incidences of myelosuppression and viral infection were lower in the LDR group than the other cases.

One serious complication associated with rituximab use is the reactivation of hepatitis $\mathrm{B}$ virus, and once reactivated, HBV may lead to death due to hepatitis in some patients. Rituximab alone without chemotherapy can induce HBV reactivation.

Yang et al. [5] also reported that rituximab increases the chance of $\mathrm{HBV}$ reactivation more than chemotherapy alone, and identified risk factors for $\mathrm{HBV}$ reactivation were being male, a lack of anti-HBs antibodies, HBV-DNA level, presence of lymphomas, anthracycline/steroid use, second-/ third-line anticancer treatment, and youth.

Huang et al. [6] reported that the lack of entecavir administration is the most important factor of $\mathrm{HBV}$ reactivation in rituximab-associated therapy. This report concluded that the most important treatment to prevent HBV reactivation was the prophylactic administration of nucleoside analog therapy, not only for $\mathrm{HBe}$ antigen (-), HBs antigen (-), and anti-HBc-positive cases but also for anti-HBs-positive cases.

By the same token, Loomba et al. [7] reported that preventive nucleoside analog approaches as lamivudine or entecavir administration are recommended when a combination of lamivudine and chemotherapy has been suggested.

Patients with so-called "resolved hepatitis B virus infection" (HBsAg-negative/cAb-positive) may also be at risk. Mozessohn et al. [8] performed a systematic review of the English and Chinese language literature to estimate the risk of hepatitis B viral reactivation in HBsAg-negative/ cAb-positive patients receiving rituximab for lymphoma. Data from 578 patients in 15 studies were included. "Clinical HBV reactivation" was estimated at $6.3 \%(\mathrm{I}(2)=63 \%, P=0.006)$. Significant heterogeneity was detected. Reactivation rates were higher in prospective vs. retrospective studies (14.2\% vs. 3.8\%; OR $=4.39$, 95\% CI 0.83-23.28). Exploratory analyses found no effect of HBsAb status on reactivation risk $(\mathrm{OR}=0.083 ; P=0.151)$. Their meta-analysis confirms a measurable and potentially substantial risk of $\mathrm{HBV}$ reactivation in HBsAg-negative/ $\mathrm{cAb}$-positive patients exposed to rituximab. Preemptive use of nucleoside analogues such as lamivudine can largely prevent HBV reactivation in patients with chronic HBV.

Lee et al. [9] analyzed the risk of HBV reactivation in hepatitis $B$ surface antigen (HBsAg)-negative/hepatitis B core antibody (anti-HBc)-positive kidney transplant patients receiving rituximab desensitization. Patients were divided into rituximab $(n=49)$ or control ( $n=123$ ) groups. All patients were observed for $\mathrm{HBV}$ reactivation, which was defined as the reappearance of hepatitis B surface antigen or HBV DNA. During the follow-up period (median, 58 months; range, 4-95 months), five patients $(10.2 \%)$ in the rituximab group and two patients $(1.6 \%)$ in the control group experienced HBV reactivation $(P=0.003)$. In the rituximab group, two patients experienced HBV-related severe 
hepatitis, and one patient died due to hepatic failure. The median time from rituximab desensitization to $\mathrm{HBV}$ reactivation was 11 months (range, 5-22 months). By contrast, no patients in the control group experienced severe hepatitis. The status of hepatitis B surface antibody was similar between groups. Rituximab desensitization [hazard ratio (HR), 9.18; 95\% confidence interval (CI), 1.74-48.86; $P=0.009$ ] and hepatitis B surface antibody status (HR 4.74; 95\% CI 1.0521.23, $P=0.04$ ) were significant risk factors for HBV reactivation. Rituximab desensitization for incompatible kidney transplantation significantly increased the risk of $\mathrm{HBV}$ reactivation in $\mathrm{HBsAg}$ negative/anti-HBc-positive patients. Therefore, close monitoring of HBV DNA is required in these patients.

B cell depleting anti-CD20 monoclonal antibody therapies are being increasingly used as long-term maintenance therapy for neuroinflammatory disease compared to many nonneurological diseases where they are used as remission-inducing agents. While hypogammaglobulinemia is known to occur in over half of patients treated with medium to long-term B celldepleting therapy (in our cohort IgG 38, IgM 56 and IgA 18\%), the risk of infections it poses seems to be under-recognized. Tallantyre [10] report five cases of serious infections associated with hypogammaglobulinemia occurring in patients receiving rituximab for neuromyelitis optica spectrum disorders.

Healy [11] reported a case of myofasciitis and meningitis with deafness caused by systemic enterovirus infection in the setting of hypogammaglobulinemia induced by rituximab.

A biopsy of the left vastus lateralis showed macrophages infiltrating the epimysium and perimysial septa, with accompanying perivascular lymphocytic cuffs. The pathology was of myofasciitis. PCR was performed on the serum and CSF, demonstrating high copy numbers of enterovirus, sequenced as echovirus type 9 .

Shah [12] also observed the low immunoglobulin $(\mathrm{Ig})$ levels in rituximab treatment.

Their cohort of 30 patients had a mean age of 63 (SD 7) years, 23 were women, 16 had granulomatosis with polyangiitis and 13 were
PR3 ANCA positive. Nine patients received concomitant cyclophosphamide. In this cohort, 20 patients had low serum IgG levels ( $<750 \mathrm{mg}$ ) dL) following RTX treatment. During the follow-up period, four individuals developed infections requiring hospitalization. In unadjusted logistic regression analysis, an $\mathrm{IgG}$ level $\leq 375 \mathrm{mg} / \mathrm{dL}$ was associated with 23 times higher odds of hospitalized infection. Low $\operatorname{Ig}$ A was also associated with an increased risk of infections requiring hospitalization after adjusting for age, race, and eGFR [OR 24.6 (95\% CI 1.5-799.5) $P=0.03$ ]. Low IgM was not associated with a higher risk of infections requiring hospitalization.

Severe hypogammaglobulinemia was associated with increased odds of infection requiring hospitalization in this cohort. Further investigation is warranted given their study is limited by small sample size, concomitant cyclophosphamide use, and variable timing of $\mathrm{Ig}$ measurement.

The effectiveness of desensitization with rituximab in ABO-incompatible renal transplantation (ABO-I) has been widely reported. However, ABO-I outcomes are still worse than those of ABO-identical or ABO-compatible renal transplantation.

Okada [13] reported ABO-I cases treated with rituximab (RIT, $n=131$ ), splenectomy (SPX, $n=21$, or neither because of low anti-A/B antibody titers (NoR/S, $n=53$ ). Graft survival, infection, and de novo HLA antibody production were compared for ABO-I and ABO-Id/C, followed by stratification into RIT and NoR/S groups. Propensity score-based methods were employed to limit selection bias and potential confounders. Overall graft survival for ABO-I was significantly lower than that for ABO-Id/C. Graft loss due to infection with ABO-I was significantly more frequent than that with $\mathrm{ABO}-\mathrm{Id} / \mathrm{C}$, whereas acute antibody-mediated rejection (AMR) caused no graft failure in ABO-I recipients. Stratified analysis demonstrated significantly higher infection risk with RIT than with NoR/S. Safe reduction or avoidance of rituximab in desensitization protocols might contribute to further improvement of ABO-I outcome. 
Morath et al. [14] observed a higher incidence of BK virus replication and BK virusassociated nephropathy. A higher frequency of viral infections such as CMV, HSV, VZV, and BK virus, as well as $P$. jirovecii pneumonia, wound, and severe urinary tract infections have been described. In the CTS and the Heidelberg cohort, an increased risk for early severe infections was observed, resulting in approximately one additional patient death in 100 ABOi kidney transplant recipients during the first year after surgery.

Transplantation in the presence of major $\mathrm{ABO}$ incompatibility, however, places the patient at a somewhat higher risk of early rejection, infection, and infection-associated death. Therefore, whenever possible, $\mathrm{ABOc}$ procedures should be preferred.

In Morath's report [15], 20 patient deaths were recorded in living donor kidney transplant recipients, with a $3.2 \%$ death rate (one of 31 ) in $\mathrm{ABO}$ and HLA incompatible kidney transplant recipients, a $1.4 \%$ rate (two of 144) in HLA incompatible patients and a $2.4 \%$ rate (six of 248) in $\mathrm{ABO}$ incompatible patients; these rates are higher than the $0.7 \%$ rate $(11$ of 1,541$)$ in standard risk recipients. The increased death rate in their cohort of desensitized patients was most likely explainable by the higher rate of infection-associated death. Although the numbers in each group are small, more than $80 \%$ of patient deaths in the $\mathrm{ABO}$ and HLA incompatible, groups were due to infection, whereas infection was the cause in only $27.3 \%$ of the deaths in standard risk recipients. In the multivariate analysis, pretransplant desensitization with plasmapheresis and rituximab was identified as the driving cause of infection-associated death (HR $=3.40, P=0.002)$.

Habicht [16] retrospectively analyzed 21 consecutive recipients who underwent ABOi renal transplantation. Pretransplant desensitization included administration of rituximab $(375 \mathrm{mg} /$ $\mathrm{m}^{2}$ ), mycophenolate mofetil (MMF), tacrolimus, and prednisolone 4 weeks prior to scheduled transplantation as well as IA and IVIG. The intensified desensitization was associated with an increased risk of infectious complications. This observation prompted them to briefly escalate the desensitization protocol in ABOi kidney recipients in their centre.

A multivariate analysis revealed that infections including viral [CMV, HSV, VZV, and polyomavirus (BKV)], as well as bacterial infections leading to hospitalization such as urosepsis and pneumonia were significantly more common in the ABOi group (50\%) than in the ABOc group (21\%) $(P=0.038)$. CMV infections occurred in $14 \%$ of ABOi recipients as compared to $6.3 \%$ of $\mathrm{ABOc}$ recipients. In the $\mathrm{ABOi}$ group, 3 of 21 patients developed HSV or VZV eruptions while only $2 \mathrm{HSV}$ infections were noted in $47 \mathrm{ABOc}$ recipients despite a similar seroprevalence of $>95 \%$ for HSV IgG and VZV IgG pretransplant in both groups. Interestingly, the most common viral infection was $\mathrm{BK}$ viremia leading to $\mathrm{BKN}$ in 5 of 20 ABOi grafts (25\%) and 4 of 47 (8.5\%) ABOc grafts.

In Lentine report [17], recipients of $\mathrm{ABOi}$ transplants experienced significantly $(P<0.05)$ higher incidence of wound infections $(12.7 \%$ vs. $7.3 \%$ ), pneumonia ( $7.6 \%$ vs. $3.8 \%$ ), and urinary tract infections (UTIs) or pyelonephritis $(24.5 \%$ vs. $15.3 \%$ ) in the first 90 days compared with ABO-compatible recipients. In adjusted models, $\mathrm{ABO}$ incompatibility was associated with twice the risk of pneumonia (adjusted hazard ratio [aHR], 2.22; 95\% confidence interval [CI], 1.144.33 ) and $56 \%$ higher risk of UTIs or pyelonephritis (aHR 1.56; 95\% CI 1.05-2.30) in the first 90 posttransplantation days, and 3.5 times the relative risk of wound infections in days 91 to 365 (aHR 3.55; 95\% CI 1.92-6.57). ABOi recipients, $19 \%$ of whom underwent pre- or peritransplant splenectomy, experienced twice the adjusted risk of early hemorrhage. ABOi transplantation offers patients with potential live donors an additional transplant option but with higher risks of infectious and hemorrhagic complications. Awareness of these complications may help improve protocols for the management of ABOi transplantation.

The single-center retrospective study by Naciri [18] assessed posttransplantation complications in $44 \mathrm{ABO}-\mathrm{i}$ versus 44 matched ABO-c patients. All patients were comparable at baseline except that ABO-i patients had greater immuno- 
logical risks. ABOi patients have a significantly increased incidence of BK-virus (BKV) infection, as well as BKV-associated nephropathy.

Infection-related mortality following desensitization with rituximab is a problem. Reduced death-censored graft survival was reported in patients who were transplanted after desensitization for HLA antibodies, most likely as a consequence of an increased rate of antibody-mediated rejection episodes. Opelz [19] suggests that patient survival after ABO (and HLA) incompatible kidney transplantations is reduced due to infectious complications and infection associated death.

Higher rates of infection-associated deaths have also been identified in several other recent studies: Barnett and colleagues [20] reported on reduced patient survival 1 and 3 years after $A B O$ incompatible kidney transplantation with four deaths in 62 desensitized patients (log rank $P=0.018$ for patient survival) [1]. One patient death was due to sepsis, while the other three patient deaths were attributable to Pneumocystis jirovecii pneumonia $(\mathrm{PcP})$.

\section{AMC Case of Hepatitis in Anti-CD20}

In our single center (Asan Medical Center) analysis [21-23], ABOi KT was performed In 500 patients and a total 152 patients underwent FCXM positive KT. A total of 65 patients who underwent ABOi and FCXM positive KT were excluded. The remaining 435 patients in the ABOi KT group were divided into era 1 (20092012) and era 2 (2012-2018) by desensitization protocol. A total of 1,209 patients who underwent $\mathrm{ABO}$ compatible and FCXM negative KT from January 2012 to February 2018 served as a control group. In our initial desensitization protocol (era 1), patients received a single dose of rituximab $(500 \mathrm{mg}) \quad 1$ week before plasmapheresis.

After we experienced lethal infectious complications, a modified immunosuppression protocol was applied from January 2012 (era 2). We reduced the dose of rituximab from 500 to $200 \mathrm{mg}$ in ABOi patients unless patients showed positive FCXM. Tacrolimus was given at an initial level of $8 \mathrm{ng} / \mathrm{mL}$ and reduced to $3-8 \mathrm{ng} / \mathrm{mL}$
1 week after transplantation. The dose of MMF was reduced from 1.5 to $1 \mathrm{~g} /$ day after the 7 th postoperative day.

The subgroup analysis of ABOi KT comprised the control group of 1,209 , the era 1 group of 64 , and the era 2 group of 371. All patients underwent ABOi KT under the condition that the $\mathrm{IgG}$ and $\operatorname{IgM~Ab}$ titers against blood groups $\mathrm{A}$ or $\mathrm{B}$ were below 1:8.

The group of patients in the era 1 showed a significantly decreased patient survival in comparison with the era 2 and control groups during the 5-year follow-up (era 1 vs. era 2 vs. control group; $92.4 \%$ vs. $98.2 \%$ vs. $99.0 \%, P<0.01$ ) (Fig. 6.1). The overall graft survival rates in era1 group showed inferior outcomes than era 2 or control group at 5-year follow-up (era1 vs. era 2 vs. control group; $89.3 \%$ vs. $95.4 \%$ vs. $96.9 \%$, $P=0.03)$.

To evaluate infectious complications according to rituximab dose, 303 patients who received an ABO i KT in the period from February 2009 to July 2016 in our center were retrospectively reviewed. The patients in era 1 showed a higher rate of infectious complications, such as CMV infection and pneumonia, compared to the patients in the era 2 or control groups (Table 6.1).

Recent years have seen increasing use of rituximab (RTX) for various types of primary and secondary glomerulopathies. However, there are no studies that specifically address the risk of infection related to this agent in patients with these conditions.

Trivin et al. [24] reviewed the outcomes of all patients who received RTX therapy for glomerular disease between June 2000 and October 2011 in eight French nephrology departments. Among 98 patients treated with RTX, 25 presented with at least one infection. They report an infection rate of 21.6 per 100 patient-years. Five patients died within 12 months following an RTX infusion, of whom four also presented with an infection. The median interval between the last RTX infusion and the first infectious episode was 2.1 months (interquartile range 0.5-5.1). Most infections were bacterial (79\%) and pneumonia was the most frequent infection reported (27\%). The presence of diabetes mellitus $(P=0.006)$, the 

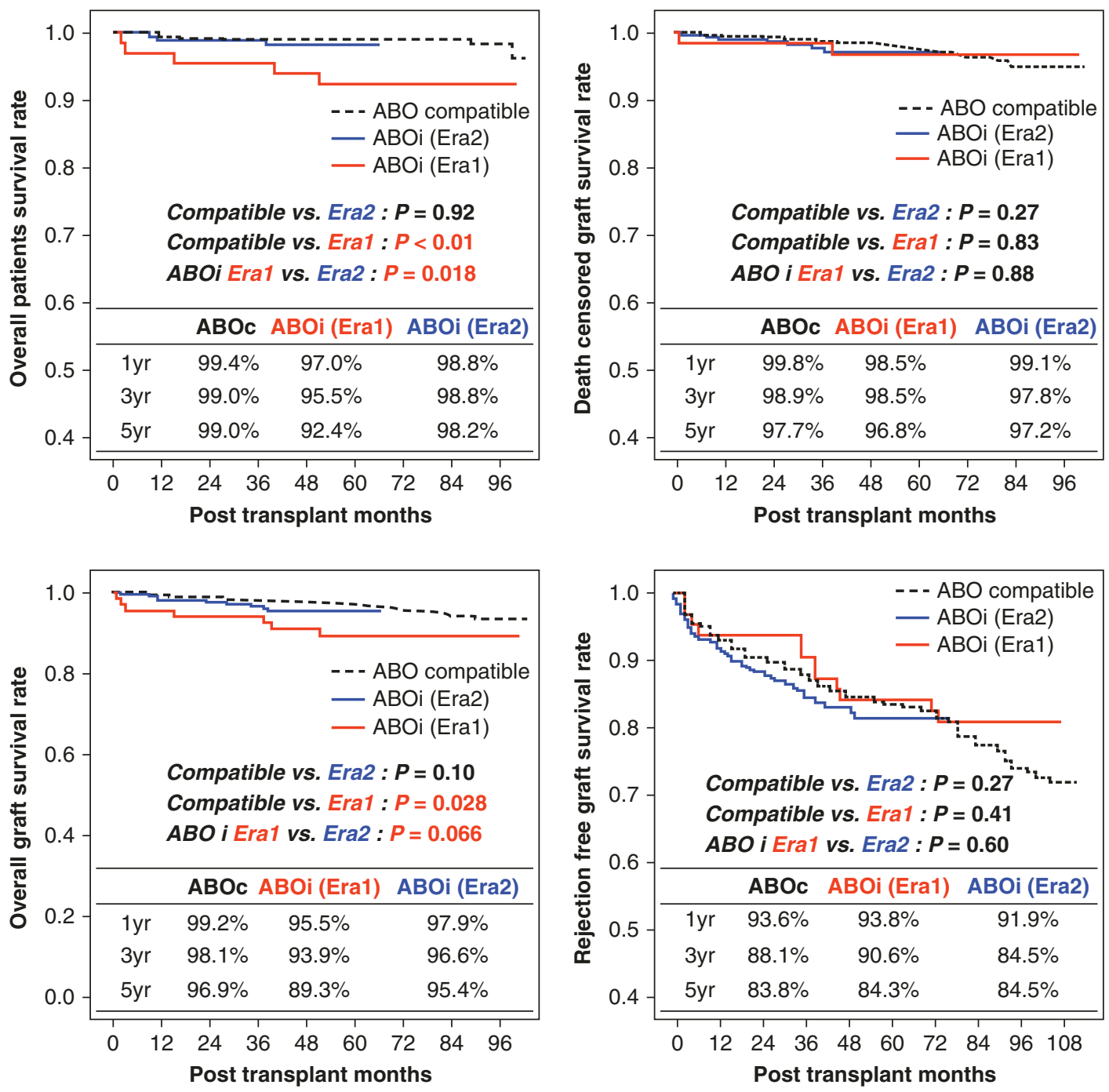

Fig. 6.1 Long-term patient and graft survival stratified by Era and ABO incompatibility

cumulative RTX dose $(P=0.01)$, and the concomitant use of azathioprine $(P=0.03)$ were identified as independent risk factors. Renal failure was significantly associated with an increased infection risk by bivariate analysis $(P=0.03)$ and was almost significant by multivariate analysis $(P=0.05)$.

Schrezenmeier [25] reported a retrospective observational registry study (German Registry on Autoimmune Diseases) comprising a total of 681 patients was conducted. The data of 63 adult kidney transplant recipients who received rituximab between 2006 and 2013 were used in this analy- sis. At least one severe infection occurred in 57\% of patients. The median time between the first rituximab infusion and the first infection was 4 (1-48) months. Of the overall 88 infections, 74 were severe bacterial infections, 5 were severe viral infections, 3 were severe fungal infections, 2 were combined severe bacterial and fungal infections, and 4 were combined severe viral, fungal, and bacterial infections. Seven patients died during the observational period, two of them due to infectious complications. In the observational period, one case of squamous cell carcinoma but no other malignancies were observed. 
Table 6.1 List of infections between ABO compatible and $\mathrm{ABO}$ incompatible KT

\begin{tabular}{|c|c|c|c|c|}
\hline \multirow[b]{2}{*}{ Variables } & $\begin{array}{l}\text { ABO } \\
\text { compatible } \\
(N=1,019)\end{array}$ & $\begin{array}{l}\text { ABOi Era } 2 \\
(N=239)\end{array}$ & $\begin{array}{l}\text { ABOi Era } 1 \\
(N=64)\end{array}$ & \multirow[b]{2}{*}{$p$-Value } \\
\hline & Number $(\%)$ & $\begin{array}{l}\text { Number } \\
(\%)\end{array}$ & Number (\%) & \\
\hline BK virus PCR- positive & $243(23.8)$ & $62(25.9)$ & $23(36.9)$ & 0.09 \\
\hline BK virus $\geq 4 \operatorname{logs}$ & $86(8.4)$ & $27(11.3)$ & $10(15.6)$ & 0.08 \\
\hline BK nephropathy & $11(1.1)$ & $8(3.3)$ & $3(4.7)$ & 0.007 \\
\hline CMV antigenemia positive & $376(36.9)$ & $72(30.1)$ & $41(64.1)$ & $<0.001$ \\
\hline $\mathrm{CMV}$ antigenemia $\geq 50$ & $88(8.6)$ & $11(4.6)$ & $11(17.2)$ & 0.004 \\
\hline Bacterial pneumonia & $40(3.9)$ & $17(7.1)$ & $2(3.1)$ & 0.09 \\
\hline PCP pneumonia & $11(1.1)$ & $0(0.0)$ & $3(4.7)$ & 0.005 \\
\hline Viral pneumonia & $13(1.3)$ & $3(1.3)$ & $3(2.7)$ & 0.08 \\
\hline Aspergillosis pneumonia & $1(0.1 \%)$ & $0(0.0)$ & $0(0.0)$ & - \\
\hline Varicella zoster & $65(6.4 \%)$ & $\begin{array}{l}14 \\
(5.9 \%)\end{array}$ & $4(6.3 \%)$ & 0.96 \\
\hline Influenza & $17(1.7 \%)$ & $5(4.5 \%)$ & $3(4.7 \%)$ & 0.22 \\
\hline Bacterial UTI & $105(10.3 \%)$ & $\begin{array}{l}17 \\
(7.1 \%)\end{array}$ & $5(7.8 \%)$ & 0.28 \\
\hline $\begin{array}{l}\text { BK virus } \geq 4 \operatorname{logs}+\mathrm{CMV} \text { antigenemia } \\
\geq 50+\text { pneumonia }+\mathrm{UTI}+\text { other viral infection }+ \text { fungal } \\
\text { infection }\end{array}$ & $333(32.7)$ & $81(33.9)$ & $31(48.4 \%)$ & 0.035 \\
\hline
\end{tabular}

A high incidence of infections was observed after rituximab treatment in kidney transplant recipients. Most infections occurred within 6 months after rituximab initiation. With more than 3 years of follow-up, they were able to document a low incidence of secondary malignancies after rituximab with only one case in their cohort.

\section{Rejection}

Unexpectedly rituximab induced high prevalence of early acute rejection was reported by Clatworthy [26]. They conducted an open-label, randomized, controlled trial comparing rituximab, a B cell depleting, chimeric, anti-CD20 monoclonal antibody, with an anti-CD25 monoclonal antibody (daclizumab) as induction therapy in patients undergoing renal transplantation. They planned to recruit 120 patients, but the study was suspended after recruitment of the first 13 patients, owing to an excess incidence of acute cellular rejection in the rituximab group. Five of six patients (83\%) who received rituximab had an episode of biopsy-confirmed cute rejection in the first 3 months after transplantation, as compared with one of seven patients (14\%) in the daclizumab group $(P=0.01)$ (Table 6.1 and Fig. 6.1). All the episodes of rejection responded to intravenous methylprednisolone, and allograft function was similar in the two groups at 12 months. After rituximab treatment, peripheral B cells were undetectable in all patients. Serum cytokines, including tumor necrosis factor $\alpha$, interleukin-6, and interleukin-10, were increased after transplantation, as compared with baseline values.

Their findings are surprising; patients who received rituximab had a rate of acute rejection that was not only higher than the rate in the control group ( $83 \%$ vs. $14 \%$ ) but also was higher than that previously observed among patients who have not received induction therapy (35\%). One possible explanation may be that proinflammatory cytokine release associated with B cell depletion might prime antigen-presenting cells. A short-lived cytokine-release syndrome often occurs after administration of the first dose of rituximab [27].

Although B cells may enhance immune responses, some B cells have immunoregulatory properties. Similarly, depletion of immunoregulatory B cells 
may have contributed to the increased rejection in the rituximab-treated patients.

\section{Lung Disease}

Pulmonary toxicity is a rare complication of rituximab therapy. Although rituximab is relatively safe and can be administered in an outpatient setting, rituximab-associated lung disease has been reported and may cause mortality despite early detection. Typically the pulmonary toxicity occurs at around the fourth cycle of rituximab. High index of suspicion is crucial and other concurrent pathology such as infective causes should be excluded. Radiological imaging and histological confirmation should be obtained and early treatment with corticosteroid should be initiated. Patients should receive counselling regarding respiratory symptoms and possible pulmonary toxicity.

Liote et al. [28] reported that post marketing surveillance detected anaphylactic shock and acute respiratory distress syndrome, fatal in 0.04-0.07\% . Rituximab-induced lung injury can be subdivided to hyperacute early (within day 1), acute and subacute (7-21 days), and chronic (>28 days) from the last administration of rituximab.

Zaidi et al. [29] reported a 49-year-old man who was diagnosed with stage IVB plasmablastic lymphoma with jaundice, night sweats, and bilateral cervical swelling for 3 weeks.

Bilateral cervical lymphadenopathy, autoimmune hemolytic anemia with left internal jugular and brachial vein thrombosis were detected and his chest $\mathrm{CT}$ was normal. He received two cycles of R-hyperCVAD (rituximab, cytoxan, doxorubicin, vincristine, dexamethasone) and two cycles of R-CHOP (rituximab, cytoxan, doxorubicin, vincristine, prednisolone) with intrathecal chemoprophylaxis during every cycle. One week prior to the fourth cycle he developed type 1 respiratory failure. On day seven post fourth cycle R-CHOP, he developed neutropenic sepsis with neutropenia. On day 14 he complained of difficulty in breathing especially on talking and exertion. Chest showed bilateral opacities. Initial bronchoalveolar lavage were negative. Therefore the most likely cause for the acute respiratory distress syndrome (ARDS) was rituximabinduced lung disease. And he later succumbed in the intensive care unit. This patient fits into the subacute onset group, which is known to be the commonest form, usually occurring around the fourth cycle of rituximab. The underlying process probably reflects hypersensitivity reaction to the immunogenic chimeric anti-CD20 antibody. Common radiological findings in rituximabinduced lung injury are diffuse pulmonary infiltrates, ground glass opacification, pulmonary fibrosis, alveolar hemorrhage, and consolidative changes.

In Hadjnicolaou's [30] report, a total of 121 cases of potential rituximab (RTX) associated interstitial lung disease (ILD) were identified from 21 clinical studies/trials, 30 case reports, and 10 cases series. The most common indication for RTX was diffuse large B cell lymphoma. RTX-ILD occurred more frequently in male patients and was most common during the fifth and sixth decades of life. In most cases, RTX was part of combination chemotherapy, but in 30 $(24.7 \%)$ cases it was given as monotherapy. The mean and median number of cycles of RTX before disease onset was four, but cases following the first cycle or as late as the 12th cycle were also identified. The mean time of onset, from the last RTX infusion until symptom development or relevant abnormal radiological change was 30 days (range 0-158 days). Abnormal radiological findings were similar in all patients, with diffuse bilateral lung infiltrates apparent on chest radiographs and/or thoracic CT. Hypoxemia was seen in all cases and pulmonary function tests were uniformly abnormal with a characteristic diffusion capacity deficit and restrictive ventilatory pattern. RTX-ILD was fatal in 18 cases.

ILD is a rare but potentially fatal complication of RTX therapy. This diagnosis should be considered in any patient who develops respiratory symptoms or new radiographic changes while receiving this biologic agent.

Child [31] report the case of an 84-year-old man with refractory immune thrombocytopenia 
purpura (ITP) who was treated with rituximab and subsequently developed severe interstitial lung disease. There has been increasing use of rituximab in the treatment of ITP with success rates of up to $62 \%$ in adult patients with recurrent ITP. Interstitial lung disease is a rare but recognized complication of rituximab but has been rarely reported in the setting of ITP.

Aquaporin-4 immunoglobulin G (IgG)positive neuromyelitis optica spectrum disorder (NMOSD-AQP4) is an inflammatory disease of the central nervous system (CNS) that predominantly affects the optic nerves and spinal cord.

Rituximab (RTX) — a monoclonal antibody to CD20 in B cells-is effective in the treatment of NMOSD. However, interstitial lung disease (ILD) is a very rare yet potentially fatal complication of RTX treatment. Ahn [32] detail the first reported case of RTX-induced ILD in a patient with NMOSD-AQP4. ILD should be suspected in patients with NMOSD undergoing RTX treatment who present with dyspnea and/or cough without any signs or symptoms of infection. Common side effects of RTX include infusionrelated reactions, fever/chills, infection, and respiratory complications.

\section{Cardiac Disease}

Life-threatening cardiac complication is reported but quite rarely.

A 62-year-old male underwent treatment of non Hodgkin's lymphoma with bone marrow involvement with thrombocytopenia was reported by Verma [33]. After 15 min of starting of IV infusion of rituximab, he started having severe retrosternal chest pain, diagnosed as acute ST elevation from inferior wall MI. Patient was preloaded with dual anti-platelets. Coronary angiogram showed $100 \%$ occlusion of proximal RCA. Thrombosuction of this culprit right coronary artery revealed underlying 90\% stenosis. After that, balloon angioplasty of RCA was done. The procedure was terminated in the view of successful balloon angioplasty with good flow. He was kept on dual antiplatelet therapy for 1 month with regular platelet monitoring. With the grow- ing increasing global use of rituximab for various oncological and immunological diseases, this complication of myocardial infarction should be kept in mind. They recommend that patients with known cardiovascular risk factors should be assessed for ischemic heart disease before treatment and be carefully monitored during and after treatment especially during first infusion when tumor burden is highest with a slow initial infusion rate, followed by increasing the rate in 30-min increments as tolerated. This lifethreatening cardiovascular complication should also be kept in mind while using rituximab in patients without cardiovascular risk factors. The mechanism by which rituximab elicits infusion reactions remains unclear, although the symptoms associated with the reactions are thought to be related to the release of inflammatory cytokines. The incidence of infusion reactions was highest during the first infusion $(77 \%)$ and decreased with each subsequent infusion. Adverse events can include urticaria, hypotension, angioedema, hypoxia, pulmonary infiltrates, acute respiratory distress syndrome, myocardial infarction, ventricular fibrillation, or cardiogenic shock. The majority of severe reactions occur approximately 30-120 min after starting the first infusion.

\section{Others}

\section{Acute Thrombocytopenia}

Rituximab-induced acute thrombocytopenia (RIAT), a rare complication of rituximab administration, is reported by Ureshino [34]. A 65-yearold man received rituximab for the treatment of high tumor burden follicular lymphoma in the leukemic phase. The next day, his platelet count abruptly dropped from 85,000 to $5,000 / \mu \mathrm{L}$, which spontaneously recovered in a few days without specific treatment. They speculate that the occurrence of infusion-related cytokine release syndrome in rituximab-sensitive high tumor burden follicular lymphoma contributed to the development of RIAT. The patient is scheduled to receive cyclophosphamide (CHOP) chemotherapy $(750 \mathrm{mg}$ / $\mathrm{m}^{2}$ cyclophosphamide, $50 \mathrm{mg} / \mathrm{m}^{2}$ doxorubicin and 
$1.4 \mathrm{mg} / \mathrm{m}^{2}$ vincristine on day 1 , and $1 \mathrm{mg} / \mathrm{kg}$ prednisolone on days 1-5) followed by rituximab. On day 19 of CHOP chemotherapy, the patient received rituximab $375 \mathrm{mg} / \mathrm{m}^{2}$ intravenously over $4 \mathrm{~h}$. Within $2 \mathrm{~h}$, he developed infusion-related hypersensitivity reactions consisting of a fever, chill, and nausea, which were resolved by $125 \mathrm{mg}$ methylprednisolone. The next day (day 20), his platelet count abruptly dropped to $5,000 / \mu \mathrm{L}$, which was verified on a peripheral smear and repeat complete blood count. The WBC also dropped to $2,200 / \mu \mathrm{L}$.

\section{Complication from Bortezomib}

\section{Q: 1. What kind of side effects does bort- ezomib have?}

2. Are the side effects related with the dose of bortezomib?

Proteasomes are located in the cell nucleus and cytoplasm and are the primary proteolytic mechanism in eukaryotic cells. The $26 \mathrm{~S}$ proteasome specifically degrades ubiquitinated proteins and eliminates unnecessary, misfolded, and malfunctioning proteins, in addition to proteins involved in cell cycle regulation and oncogenesis. Bortezomib, a modified dipeptidyl boronic acid, is a reversible inhibitor of the $26 \mathrm{~S}$ proteasome. Its ability to trigger apoptosis of bone marrowderived plasma cells has led to its approved and widespread use in multiple myeloma. Bortezomib also shows several additional immunological effects that have resulted in its increasing utility in AMR and desensitization protocols. Bortezomib mediates apoptosis of activated $\mathrm{T}$ ells by preventing degradation of IkB and blocking nuclear factor-kB and subsequent transcription of interleukin-1, interleukin-6, and tumor necrosis factor-a. Specifically, bortezomib induces the loss of mitochondrial membrane potential, which translocates proapoptotic proteins and enhances caspase activities. Bortezomib suppresses the maturation of dendritic cells, which present antigens to $T$ cells including the HLA antigens. Bortezomib inhibits angiogenesis by reducing VEGF and interleukin-release by tumor cells and prevents adaptation to hypoxia. In chronic allograft injury, a lymphangiogenesis process is involved so this inhibition could further disrupt rejection from taking place [35]. Bortezomib is a proteasome inhibitor that downregulates the $\mathrm{T}$ cell immune response and is increasingly being used for kidney transplant rejection.

\section{GI Toxicity}

Prospective open labeled clinical trial using bortezomib in sensitized renal transplant was performed by Jeong [36]. The desensitization regimen consisted of 2 doses of IVIG ( $2 \mathrm{~g} / \mathrm{kg})$, a single dose of rituximab and 4 doses of bortezomib. There were 19 highly sensitized patients who received desensitization and 17 patients in the control. Deceased donor kidney transplantation was successfully performed in 8 patients $(42.1 \%)$ in the desensitization group versus 4 $(23.5 \%)$ in the control group. Desensitization was well tolerated, and acute rejection occurred only in the control group. Desensitization protocol using bortezomib, high-dose IVIg, and rituximab increased the DDKT rate in highly sensitized, wait-listed patients. One patient received only two doses of bortezomib because of neutropenia and abdominal pain after the second dose of bortezomib. Regarding adverse events, gastrointestinal toxicity was the most common adverse event (21\%), followed by opportunistic infection (15.8\%), and thrombocytopenia.

\section{Peripheral Neuropathy}

Based on the few clinical trials that investigate its safety and efficacy, bortezomib appears to be successful as a desensitization agent. Notably, Shah [37] observed that $50 \%$ of the patients on bortezomib experienced a worsening or increase 
in peripheral neuropathy during the first year of transplantation, although no severe cases were reported.

Gonzales [38] suggest that 32 doses of bortezomib monotherapy was not well tolerated and resulted in only a modest reduction in anti-HLA antibodies. Bortezomib was given in cycles (4 doses $=1$ cycle). The initial dose for all patients was $1.3 \mathrm{mg} / \mathrm{m}^{2}$ body surface area (BSA) intravenously on days $1,4,8$, and 11 with at least 10 days in between the last dose of a cycle and the first dose of the next cycle. Five patients (50\%) completed the 32-dose regimen without dose reduction or discontinuation. Dose reduction was required in two patients who developed severe peripheral neuropathy but eventually completed the 32-dose course. The severe neuropathy was manifested by left scapular myofascial pain and upper extremity edema. The other patient experienced progressive severe bilateral lower extremity neuropathy, anorexia, and insomnia. The other patient developed disseminated varicella zoster, severe local herpes recurrence, peritonitis in the setting of peritoneal dialysis, encephalopathy, ataxia, and visual hallucinations.

\section{Hematologic Toxicity}

No systemic trial has been undertaken to support its use in ABMR. In randomized, placebocontrolled trial (the Bortezomib in Late AntibodyMediated Kidney Transplant Rejection [BORTEJECT] Trial), Eskandari [39, 40] investigated whether two cycles of bortezomib (each cycle: $1.3 \mathrm{mg} / \mathrm{m}^{2}$ intravenously on days $1,4,8$, and 11) prevent GFR decline by halting the progression of late donor-specific antibody (DSA) positive ABMR. Forty-four DSA positive kidney transplant recipients with characteristic ABMR morphology. Patients were randomly assigned to receive bortezomib $(n=21)$ or placebo $(n=23)$. They detected no significant differences between bortezomib- and placebo-treated groups in median measured GFR at 24 months, 2-year graft survival $(81 \%$ vs. $96 \% ; P=0.12)$, urinary protein concentration, DSA levels, or morphologic or molecular rejection phenotypes in 24-month follow-up biopsy specimens. Bortezomib, however, associated with gastrointestinal and hematologic toxicity. The grades of anemia, thrombocytopenia, and leukocytopenia were significantly higher in the bortezomib group.

\section{Compensatory Humoral Reaction}

In Kwun study [41] bortezomib treatment given intravenously twice weekly for 1 month $\left(1.3 \mathrm{mg} / \mathrm{m}^{2}\right.$ per dose) clearly reduced the numbers of antibody-producing cells and CD38 + CD19 + CD20 - plasma cells in the bone marrow $(P<0.05)$, but donor-specific alloantibody levels did not decrease. They observed a rapid but transient induction of circulating IgG + B cells and an increased number of proliferating B cells in the lymph nodes after 1 month of treatment. Notably, bortezomib treatment induced germinal center B cell and follicular helper $\mathrm{T}$ cell expansion in the lymph nodes. These data suggest that bortezomib-induced plasma cell depletion triggers humoral compensation. On the basis of the effect of bortezomib on PC and in vivo compensation, they suggest that bortezomib should not be used alone for desensitization, because bortezomib depletion of PCs will lead to a compensatory response, which is likely to be deleterious to an allograft. They suggest that desensitization regimens incorporating the unique effect of bortezomib should be designed on the basis of its mechanistic effects and limitations and that adjuvant B cell depletion, blocking of GC initiation, or strategies to prevent GC compensation should be considered.

\section{Others}

In a study of bortezomib in a new player in preand posttransplant desensitization [42], mean drug-related adverse events that were reported in more than $15 \%$ of patients enrolled in those studies consisted of asthenic condition (fatigue, weakness, and malaise), GI disorders, pyrexia, 
thrombocytopenia, neutropenia, peripheral neuropathy, psychiatric disorders, and anorexia.

\section{Complication from IVIg}

\section{Q: 1. What kind of side effect doses IVIg have? \\ 2. Is it related with the dose of IVIg injection?}

Intravenous Ig (IVIg) was initially used to treat primary immune deficiencies and then for the treatment of various autoimmune, inflammatory, and infectious diseases.

Intravenous IVIg is applied to desensitize highly HLA-sensitized patients and to treat antibody mediated rejection (AMR). It is also used in the treatment of polyomavirus and parvovirus disease.

Some common side effects of IVIg infusion include pyrexia, rigors, and headache. Rare, but significant, adverse events include acute kidney injury related to sucrose-induced osmotic nephrotoxicity, hypersensitivity reaction, and vascular thrombosis [43].

\section{Thromboses}

It is reported that life-threatening thromboses in pulmonary, coronary, cerebral, and peripheral vessels are associated with high-dose intravenous immunoglobulin (IVIg) therapy that is generally considered safe [44].

Sin [45] experienced a patient with a renal graft rupture that developed after high-dose IVIg was administered for desensitization. A needle biopsy performed 4 days prior to the rupture revealed the presence of glomerular thrombosis and mesangiolysis. The ruptured nephrectomy specimen contained renal infarction around the hemorrhagic segment and arterial wall thickening with intimal fibrosis. This might have contributed to rupturing associated with small arterial and glomerular arteriolar thrombi. The mechanisms underlying thrombosis development are IVIg-induced platelet activation, increased plasma viscosity, and coagulation factor XI contamination. She also received two rounds of high-dose IVIg $(1 \mathrm{~g} / \mathrm{kg}$ per day for 2 days) due to $100 \%$ PRA (panel reactive antibody) against class I and 92\% against class I IHLA antigens as well as positive crossmatch test results against $\mathrm{T}$ cells. Fourteen days after surgery, IVIg was administered at a dose of $1 \mathrm{~g} / \mathrm{kg}$ per day for 2 days to further reduce. Two days later, the creatinine levels had increased to $2.2 \mathrm{mg} / \mathrm{dL}$. A biopsy showed that thromboembolisms had formed in the glomeruli along with focal segmental mesangiolysis. Four days later, the patient experienced severe graft pain. At the operation field a large perirenal hematoma with graft rupture was observed. The patient subsequently underwent hemodialysis.

In general, high doses of IVIG are relatively safe. However, serious side effects have been reported including acute renal dysfunction likely related to high osmotic load, thrombotic events with rapid infusions, and aseptic meningitis [46]. Slowing the infusion rate and using iso-osmolar preparations can reduce the risk of side effects [47].

\section{Hemolysis}

Kahwaji [48] report the experience with IVIginduced hemolytic anemia (IH) in ESRD patients receiving IVIG for desensitization or treatment of AMR. High cumulative doses were administered, $\geq 2 \mathrm{~g} / \mathrm{kg}$, in most cases. Markers of hemolysis, including direct antiglobulin tests, were recorded. There were 18 cases of IH in 16 patients. All patients developing hemolysis were non-O blood types. Isohemagglutinin titers ranged from 1:2 to $1: 64$ in the various IVIg products. Acute IH is a significant complication of high-dose IVIg infusion. Identified risk factors include non-O blood type of the recipient and administration of liquid IVIg preparations with high-titer anti-A/B IgG antibodies. They recommend monitoring hemoglobin $48-72 \mathrm{~h}$ 
after IVIG infusion. If the hemoglobin decreases, a hemolytic workup is recommended. Hemolysis could be avoided in at-risk patients by choosing a low-titer product. However, other complications such as acute renal failure or thrombosis may be seen because the low-titer products are usually hyperosmotic.

Intravenous immunoglobulin (IVIg) is used for the treatment of a number of inflammatory conditions. Hemolysis due to passive transfer of blood group antibodies is a well-recognized complication of IVIg therapy. Therapy is largely supportive and consists of blood product support and hemodialysis. Welsh [49] report the use of therapeutic plasma exchange (TPE) as adjunct therapy for three patients with complications attributed to IVIg. Two patients had hemolysis attributed to IVIg; one patient was blood group A and the other blood group $\mathrm{O}$.

TPE may be useful therapy for patients with severe hemolysis caused by IVIg or at risk for tissue damage by blood group antibodies.

\section{Myocardial Infarction}

In Stenton report [50], an 81-year-old Vietnamese man was prescribed IVIG for treatment of toxic epidermal necrolysis secondary to allopurinol. Thirty minutes following the start of the IVIG infusion, the patient developed crushing retrosternal chest pain and shortness of breath. The pain improved upon discontinuation of IVIG infusion but recurred when IVIG was restarted. The troponin level reached $140 \mathrm{microg} / \mathrm{L}$, and a persantine sestamibi stress test (MIBI) indicated anterolateral ischemia. The patient was diagnosed with non-ST-elevation MI. Although an association between IVIG administration and MI has not been demonstrated in clinical trials, accumulating clinical experience suggests that a relationship between IVIG and myocardial ischemia exists. Twenty published case reports were identified. Risk of acute MI seems to be increased with use of high-dose IVIG and in older individuals, especially those with at least one cardiovascular risk factor, such as ischemic heart disease or hypertension.

\section{Others}

In Kakuta's report [51] 17 patients, each showing positive T cell FCXM (median ratio $\geq 1.4$ ) after 2 rounds of double-filtration plasmapheresis, received 4-day regimens of IVIG ( $1 \mathrm{~g} / \mathrm{kg}$ per day) over 1-week periods. T cell and B cell FCXM determinations were obtained after every IVIG dose and again up to 4 weeks after initiating IVIG to ascertain negative conversion of $\mathrm{T}$ cell FCXM (median ratio < 1.4). The T cell FCXM-negative conversion rates after cumulative doses of 1, 2, 3, and $4 \mathrm{~g} / \mathrm{kg}$ IVIG were $29.4 \%, 35.3 \%, 56.3 \%$, and $46.7 \%$, respectively. No deaths occurred during the study period. Adverse events, moderate or mild (total of 52), were observed in 13 patients $(76.5 \%)$, and side effects (total of 38 ) were recorded in 11 patients $(64.7 \%)$. The chief side effects included headache $(29.4 \%)$, hepatic dysfunction (17.6\%), rash (17.6\%), and nausea $(11.8 \%)$. Four patients $(23.5 \%)$ suffered serious adverse events (total of 6), and serious side effects (total of 5) were recorded in 3 patients (17.6\%). Serious side effects included leukopenia (5.9\%), neutropenia (5.9\%), thrombocytopenia (5.9\%), headache $(5.9 \%)$, and aggravation of renal function $(5.9 \%)$, all of which were predictable. The protocol was discontinued in 2 patients after the third dose of IVIg. One became cytopenic (leukopenia, neutropenia, thrombocytopenia), without need of granulocyte colony-stimulating factor or platelet transfusions, and the other experienced headaches. In another patient, hemodialysis was initiated for the first time and continued until the point of transplantation to address deteriorating renal function after IVIg administration. There was no thromboembolism which is a concern due to the large dose of IVIg.

\section{Complication from Eculizumab}

Q: 1. What kind of side effect does eculizumab have?

2. What kind of vaccination is needed for the prevention of lethal infection? 
The effector functions of complement are centered on the formation of the $\mathrm{C} 5$ convertases that cleave the $\mathrm{C} 5$ to $\mathrm{C} 5 \mathrm{a}$ and $\mathrm{C} 5 \mathrm{~b}$. C5a as well as $\mathrm{C} 3 \mathrm{a}$ which are anaphylatoxins involved in inflammation amplification and antigen presentation. C5b leads to formation of C5b-9 membrane attack complex, which can lyse nonnucleated cells (RBC, bacteria) or cause cellular activation and tissue injury when binding to nucleated cells. At the level of the kidney, primary dysregulation of the complement cascade is associated with thrombotic microangiopathy or C3 glomerulopathy. Eculizumab (Soliris) is a humanized monoclonal antibody, which blocks the cleavage and the activity of complement factor 5 .

Humanized monoclonal antibody that binds C5 and blocks the enzymatic effect of C5 convertase thus preventing the creation of the membrane attack complex. The blockade is predominantly due to steric hindrance. In addition to a decrease in membrane attack complex formation via $\mathrm{C} 5 \mathrm{~b}$, eculizumab blocks the release of $\mathrm{C} 5 \mathrm{a}$, a potent anaphylatoxin. C5a has been associated with the recruitment of phagocytes and upregulation of proinflammatory cytokines [52].

Clinical applications of eculizumab have been extended to AMR and prevention of delayed graft function in kidney transplantation. Duration and monitoring of treatment with eculizumab for complement defects is unclear. In AMR, it is used as a preventive measure following desensitization protocols or as salvage attempts in refractory AMR.

Limited data are available for the use of eculizumab in desensitization and AMR treatment. Its applications are complementary to desensitization protocols or to commonly used approaches for AMR treatment like plasma exchange, IVIg, and rituximab.

\section{Infection}

The largest and most systematic clinical experience with eculizumab use for prevention of AMR was reported by Cornell, Stegall $[53,54]$ in which outcomes beyond 1 year in eculizumab-treated (EC) positive crossmatch kidney transplants (+XMKTx) was compared to a historical control group. +XMKTx received desensitization with either plasma exchange (PE) alone $(N=48)$ or $\mathrm{PE}$ and $\mathrm{EC}(N=30)$. EC, given for at least 1 month, was continued in the setting of persistently high DSA (B flow cytometric crossmatch [BFXM] >200) including: 4 weeks $(n=14)$; 9 weeks $(n=6), 6$ months $(n=2)$, and 12 months $(n=8)$. All patients had at least 2 years followup. The EC dosing regimen consists of 1,200 mg immediately prior to transplantation, $600 \mathrm{mg}$ on postoperative day 1 , and $600 \mathrm{mg}$ weekly thereafter for 4 weeks.

In patients with persistently high DSA (BFXM $>200)$, EC treatment continued (1,200 mg week 5 , and then every 2 weeks). DSA assessments were performed at weeks 4, 9, 26, and 39 . Eculizumab was discontinued at those time points if the B flow crossmatch channel shift was $<200$. Thirty patients received eculizumab from June 2008 to October 2011. Forty-eight historical controls were transplanted using a similar PEand IVIG-based "desensitization."

Patients underwent induction therapy with anti-thymocyte globulin; and maintenance immunosuppression consisted of tacrolimus, mycophenolate mofetil, and prednisone.

The incidence of acute clinical ABMR was lower in the EC group than controls (6.7\% vs. $43.8 \% p<0.01)$. Death-censored allograft survival was similar between groups.

Despite decreasing acute clinical ABMR rates, eculizumab treatment does not prevent chronic ABMR in recipients with persistently high BFXM after +XMKTx.

In the eculizumab group. There was one episode of subclinical acute cellular rejection. There was one wound infection in the eculizumab group. One patient in the eculizumab group developed Burkitt's lymphoma 2.5 years after transplantation and died with a functioning graft.

West-Thielke [55] reported four patients underwent living donor kidney transplant from ABOi donors who were treated with a 9-week eculizumab course without therapeutic plasma exchange, intravenous immunoglobulin, or splenectomy. All patients had successful transplants and have normal graft function at the time of last follow-up. There were no cases of AMR or acute cellular rejection. 
Patients received vaccination against Haemophilus influenzae, Streptococcus pneumoniae, and Neisseria meningitidis. Patients were immunized with conjugated, quadrivalent meningococcal vaccine (Menactra, Sanofi Pasteur, Lyons, France; Menveo, Novartis Vaccines and Diagnos tics, Cambridge, MA) protecting against strains $\mathrm{A}, \mathrm{C}, \mathrm{Y}$, and $\mathrm{W} 135$ at least 2 weeks prior to receiving TPEX. In addition, patients were maintained on chemoprophylaxis with penicillin $500 \mathrm{mg}$ every $12 \mathrm{~h}$ (levo-floxacin was utilized in patients allergic to penicillin) starting at time of eculizumab administration and continuing for 4 weeks after therapy with eculizumab was discontinued. One patient developed nausea, shoulder pain, and chest heaviness during the infusion of eculizumab on POD 49, which required early discontinuation of therapy.

Most serious infectious concerns are related to an increased risk of infections with encapsulated bacterial strains [56], Neisseria meningitides most notable, where complement activity plays an important role in the immune responses. Lifethreatening infections with $\mathrm{N}$. meningitides have been reported, and vaccination is recommended by the Advisory Committee on Immunization Practices.

Current recommendations for adult patients receiving eculizumab treatment include two doses of MenACWY at least 2 months apart with booster vaccinations every 5 years.

MenB vaccination is now recommended as well for patients $>10$ years of age. Vaccination is recommended at least 2 weeks prior to initiation of treatment with eculizumab.

Their protocol of eculizumab use posttransplantation includes vaccination with both conjugate tetravalent and MenB vaccination and 4-8 weeks of antibiotic prophylaxis with penicillin $\mathrm{V}$ or ciprofloxacin.

\section{Others}

The efficacy of eculizumab for desensitization and treatment of AMR is unclear as complexed by patient characteristics and other therapeutic modalities [56].
Eculizumab safety data monitoring is ongoing and its availability is restricted by the FDA under a Risk Evaluation and Mitigation Strategy. Safety monitoring is ongoing; so far, most notable is the expected increase in infection risk with encapsulated organisms.

In general, eculizumab infusions are well tolerated by most patients with minimal adverse reactions noted. When adverse events occur, those commonly reported are: diarrhea, nausea, hypertension, headache, and vomiting. Additionally, anemia, leukopenia, urinary tract infections, and viral infections have been reported.

In Kulkarni report [57], 15 participants (five control, 10 treatment) with DSA and deteriorating renal function were enrolled. The treatment group received 6 months of eculizumab followed by 6 months of observation, whereas controls were observed.

The treatment group had an improved eGFR trajectory versus control, based on their predetermined two-sided 0.10 significance level $(p=0.09)$. Within-subject analysis of treated participants at 6-month intervals did not show significant change $(p=0.60)$.

Modeling C1q status showed that C1q-positive patients had significantly higher mean eGFR than patients with negative C1q $(p=0.04)$. Biopsies revealed elevated renal endothelial cell-associated transcripts (ENDATs), ENDATs in most participants, but ENDATs were not reduced with complement inhibition.

There were no significant differences in adverse events between the two arms of the study. There were no deaths or episodes of biopsyproven acute rejection.

\section{Complication from C1 Esterase Inhibitor (C1 INH)}

\section{Q: 1. What kind of side effect dose the} C1 INH have?

2. What is the difference of side effect between eculizumab and C1 INH? 
C1-INH, which is a member of the serpin family of pro-tease inhibitors, inactivates $\mathrm{C} 1 \mathrm{r}$ and $\mathrm{C} 1 \mathrm{~s}$ and is the only plasma protease that regulates the classic complement pathway.

Administration of exogenous human $\mathrm{C} 1$ esterase inhibitor $(\mathrm{C} 1 \mathrm{INH})$ may provide prolonged protection from complement-mediated damage. Recombinant $\mathrm{C} 1 \mathrm{INH}$ prevented acute $\mathrm{AMR}$ in a primate model [58]. CINRYZEâ (Shire ViroPharma Incorporated, Lexington, MA) is a human plasma-derived $\mathrm{C} 1 \mathrm{INH}$ that is approved for use in patients with hereditary angioedema.

Up until now there is not a report on the prophylactic use of $\mathrm{C} 1$ esterase inhibitor in sensitized renal transplant recipients.

\section{Infection}

Jordan et al. [59, 60] investigate the ability of $\mathrm{C} 1$ esterase inhibitor (C1INH) to prevent IRI/DGF in kidney transplant recipients. Seventy patients receiving deceased donor kidney transplants at risk for DGF were randomized to receive $\mathrm{C} 1 \mathrm{INH}$ $50 \mathrm{U} / \mathrm{kg}(n=35)$ or placebo $(n=35)$ intraoperatively and at $24 \mathrm{~h}$. The primary end point was need for hemodialysis during the first week posttransplant. Assessments of glomerular filtration rate and dialysis dependence were accomplished.

C1INH did not result in reduction of dialysis sessions at 1 week posttransplant, but significantly fewer dialysis sessions $(P=0.0232)$ were required 2-4 weeks posttransplant. Patients at highest risk for DGF (Kidney Donor Profile Index $\geq 85$ ) benefited most from C1INH therapy.

There were no differences in patient and graft survival or graft losses at 1 year. One patient was removed from study analysis due to development of a significant posttransplant bleeding that led to acute kidney injury in a well function allograft. This was not considered to be related to the C1NH. C1NHY level were significantly higher in the treatment group, and this persisted at 1 week posttransplant.

Twenty patients (28.6\%) experienced SAE. Ten from $\mathrm{C} 1 \mathrm{NH}$ and 10 from the placebo group. All SAE resolved with treatment and were deemed not to be related to $\mathrm{C} 1 \mathrm{NH}$. Additional safety concerns with $\mathrm{C} 1 \mathrm{NH}$ were risk of meningococcal infection and venous thromboembolism. At the 1-year assessment, there were two graft losses: one in the placebo group (antibody mediated rejection) and one in the $\mathrm{C} 1 \mathrm{NH}$ group (surgical complication). The graft loss in the $\mathrm{C} 1 \mathrm{NH}$ group was due to repeated retroperitoneal hematomas with acute kidney injury due to bleeding from a pseudoaneurysm of the iliac artery and renal artery anastomosis site. Significantly better renal function was seen at 1 year in C1INH patients $(P=0.006)$. No significant adverse events were noted with $\mathrm{C} 1 \mathrm{INH}$.

\section{Others}

Montgomery [61] report a phase 2b, multicenter double-blind randomized placebo-controlled pilot study to evaluate the use of human plasma-derived $\mathrm{C} 1$ esterase inhibitor $(\mathrm{C} 1 \mathrm{INH})$ as add-on therapy to standard of care for AMR. Eighteen patients received 20,000 units of C1 INH or placebo (C1 INH $n=9$, placebo $n=9$ ) in divided doses every other day for 2 weeks. No discontinuations, graft losses, deaths, or study drug-related serious adverse events occurred.

C1 INH group demonstrated a trend toward sustained improvement in renal function. Sixmonth biopsies performed in 14 subjects $(\mathrm{C} 1$ $\mathrm{INH}=7$, placebo $=7$ ) showed no transplant glomerulopathy (TG) (PTC $+\mathrm{cg} \geq 1 \mathrm{~b})$ in the C1 INH group, whereas 3 of 7 placebo subjects had TG. Endogenous C1 INH measured before and after PP demonstrated decreased functional C1 INH serum concentration by $43.3 \%$.

This new finding suggests that $\mathrm{C} 1 \mathrm{INH}$ replacement may be useful in the treatment of AMR.

Seven $(78 \%)$ of nine subjects receiving $\mathrm{C} 1$ INH and six (67\%) of nine receiving placebo had resolution of their AMR with a median time from first dose until AMR recovery of 20.0 days (range 19-86 days) in the $\mathrm{C} 1 \mathrm{INH}$ group and 20.5 days (range 20-22 days) in the 
placebo group. Among the 6-month biopsies performed, no $\mathrm{C} 1 \mathrm{INH}$ subjects showed TG (PTC $+\mathrm{cg} \geq 1 \mathrm{~b}$ ), whereas three of seven placebo subjects had TG. In the three placebotreated subjects whose biopsies showed TG, there was evidence of ongoing chronic AMR.

Among 18 patients, 15 subjects $(83 \%)$ had one or more AE during the study, which included nine subjects $(100 \%)$ in the $\mathrm{C} 1 \mathrm{INH}$ group and six subjects $(67 \%)$ in the placebo group. Only one patient $(11 \%)$ in the $\mathrm{C} 1 \mathrm{INH}$ group had an AE (mild blurred vision) that was considered by the investigator to be related to study drug.

The most frequently reported $\mathrm{AE}$ was diarrhea, reported by three $(33 \%)$ subjects in the $\mathrm{C} 1$ INH group and one $(11 \%)$ patient in the placebo group. Peripheral edema $(n=3)$, dyspepsia $(n=2)$, pruritus $(n=2)$, and urinary tract infection $(n=2)$ occurred in subjects in the $\mathrm{C} 1 \mathrm{INH}$ group but not in subjects in the placebo group.

Viglietti [62] performed a prospective, single-arm pilot study to investigate the potential effects and safety of $\mathrm{C} 1$ inhibitor (C1-INH). Berinert (C1-INH) added to high-dose intravenous immunoglobulin (IVIG) for the treatment of acute ABMR that is nonresponsive to conventional therapy. Kidney recipients with nonresponsive active ABMR and acute allograft dysfunction were enrolled between April 2013 and July 2014 and received C1-INH and IVIG for 6 months (six patients). The primary end point was the change in eGFR at 6 months after inclusion $(M+6)$. Secondary end points included the changes in histology and DSA characteristics and adverse events as evaluated Until 6M.

No death or allograft loss was observed in C1-INH patients. One serious adverse event, i.e., gastrointestinal bleeding, occurred in one patient. The event was not considered to be related to the study drug. One episode of deep venous thrombosis of a lower limb occurred 5 months after inclusion in the study (adverse event), which led to Berinert discontinuation in this patient. This episode was caused by local venous compression due to a popliteal cyst.

\section{Complication from IgG Endopeptidase (IdeS)}

\section{Q: 1. What kind of side effect does IdeS have? \\ 2. What is the rebound of DSA? \\ 3. What is the preventive measure for rebound DSA?}

The IgG-degrading enzyme derived from Streptococcus pyogenes (IdeS; GenBank accession number, ADF13,949.1) is a recombinant cysteine protease of $S$. pyogenes produced in Escherichia coli that cleaves all four human subclasses of IgG with strict specificity by hydrolyzing human IgG at Gly236 in the lower hinge region of the $\operatorname{IgG}$ heavy chains and cleaving human $\operatorname{IgG}$ into $\mathrm{F}(\mathrm{ab}$ ') 2 and $\mathrm{Fc}$ fragments inhibiting complement-dependent cytotoxicity and antibody-dependent cellular cytotoxicity, which suggests that IdeS might be useful for desensitization. IdeS also cleaves B cell receptors from circulating B cells, with the resultant inhibition of antigen specific $B$ cell $\mathrm{IgG}$ responses in vitro [63]. Within minutes after dosing, plasma $\operatorname{IgG}$ was converted into scIgG, and within a few hours after IdeS treatment, plasma IgG was cleaved into $\left.\mathrm{F}(\mathrm{ab})_{2}\right)_{2}$ and Fc fragments with no intact IgG [64].

\section{Rebound Antibody Reaction}

Jordan et al. [65] administered IdeS to 25 highly HLA-sensitized patients (11 patients in Uppsala or Stockholm, Sweden, and 14 in Los Angeles) before the transplantation of a kidney from an HLA-incompatible donor.

Patients in the US study also received intravenous immune globulin and rituximab after transplantation to prevent antibody rebound. Eligible patients were awaiting kidney transplantation on either the United Network for Organ Sharing waiting list (in the United States) or the Scandia transplant waiting list (in Sweden) with panel- 
reactive antibody level of 95\% (range, 22-100). The acceptance criteria for HLA-incompatible organs in recipients in the United States include a negative complement-cytotoxicity crossmatch, a negative flow-cytometric crossmatch, or a positive $\mathrm{T}$ cell and B cell flow-cytometric crossmatch with approximately 250 channel shifts or less and usually donor-specific antibody positivity.

In Sweden, patients were eligible if they had at least two anti-HLA antibodies with a mean fluorescence intensity of 3,000 or more.

Patients who underwent transplantation received IdeS at a dose of $0.24 \mathrm{mg}$ per kilogram of body weight (in the United States) or at a dose of 0.25 or $0.50 \mathrm{mg} / \mathrm{kg}$. Horse antithymocyte globulin is not susceptible to digestion by IdeS (unpublished data). Patients in the U.S. cohort received induction with alemtuzumab, at a dose of $30 \mathrm{mg}$ administered subcutaneously 4 days after transplantation. and received IdeS over a period of $15 \mathrm{~min}$ approximately $4-6 \mathrm{~h}$ before the receipt of a kidney transplant from an incompatible donor. By $6 \mathrm{~h}$ after the start of the infusion, all the IgG molecules are completely cleaved into $\mathrm{Fc}$ and $\mathrm{F}\left(\mathrm{ab}^{\prime}\right) 2$ fragments, which probably reduces their pathogenicity. All the $\operatorname{IgG}$ molecules are inactivated for approximately 1-2 weeks, when new IgG synthesis is detected. There was a significant reduction in the total $\mathrm{IgG}$ level that persisted for 28 days. Briefly, nearcomplete inhibition of C1q-binding HLA antibodies was seen $1 \mathrm{~h}$ after treatment. The levels of all HLA antibodies were significantly reduced at $6 \mathrm{~h}$ after treatment. Rebound occurred in Swedish. In contrast, the U.S. cohort had fewer patients with rebound and lower levels of HLA antibodies after treatment with IdeS.

One patient in the US study had hyperacute rejection immediately after revascularization. This event was unexpected, since the tests for crossmatches and donor-specific antibodies were negative after IdeS treatment and before transplantation. Extensive investigations after the rejection showed high-titer $\operatorname{IgM}$ and $\operatorname{IgA}$ antibody reactive with donor-allograft endothelium, which they speculate was probably responsible for the immediate graft loss.
Thus, the antibody appears to be a non- HLA antibody that cannot be cleaved by IdeS.

There were 13 infectious complications that generally responded to treatment. However, in the Swedish study, one patient had prolonged parvovirus B19 viremia and one had persistent myalgias after the IdeS infusion. A total of 38 serious adverse events occurred in 15 patients (five events were adjudicated as being possibly related to IdeS). At transplantation, total IgG and HLA antibodies were eliminated. A total of 24 of 25 patients had perfusion of allografts after transplantation. Antibody-mediated rejection occurred in ten patients (seven patients in the U.S. study and three in the Swedish study) at 2 weeks to 5 months after transplantation; all these patients had a response to treatment. One graft loss, mediated by non-HLA IgM and IgA antibodies, occurred. The use of intravenous immune globulin and rituximab after transplantation probably prevents rebound donor-specific-antibody responses to some extent.

A subsequent multicenter, multinational, phase 2 trial of $\mathrm{IgG}$ endopeptidase for desensitization [66] is currently ongoing (NCT02790437). The immediate effect of IgG endopeptidase on donorspecific antibodies in the preliminary study was profound, with near or complete reduction of HLA antibodies at $6 \mathrm{~h}$. Suppression of HLA antibodies was durable among patients treated with intravenous immunoglobulin and rituximab in the United States.

\section{Immunogenicity of IdeS}

Lonze et al. [67] present Phase II, single-arm open label trial to assess the efficacy of IdeS to convert a positive crossmatch test to negative prior to transplantation with either a deceased or living donor kidney namely seven highly sensitized (cPRA98-100\%) kidney transplant candidates who had DSA resulting in positive crossmatches with their donors (5 deceased, 2 living).

All pre-IdeS crossmatches were positive and would have been prohibitive for transplantation. 
All crossmatches became negative post-IdeS and the patients underwent successful transplantation.

All received IdeS as monotherapy for desensitization and underwent successful transplantation within $24 \mathrm{~h}$ of IdeS administration. IdeS $(0.25 \mathrm{mg} / \mathrm{kg}$, intravenously) was given followed by repeat crossmatch tests $2-$ and 6-h postdose. A second dose of IdeS $(0.25 \mathrm{mg} / \mathrm{kg}$, intravenously) was administered if the 2 -h crossmatch did not convert to negative. The transplant operations were performed as soon as a negative crossmatch result was obtained. In all cases, the transplant operation took place within $24 \mathrm{~h}$ of the first IdeS dose.

Induction immunosuppression consisted of high-dose corticosteroids (methylprednisolone $1,000 \mathrm{mg}$, intravenously), given in the operating room. Intravenous corticosteroids were tapered over 5 days and then converted to prednisone (30 mg, orally). Alemtuzumab (Campath, Millennium and ILEX Partners LP, Cambridge, MA, $20 \mathrm{mg}$, intravenously or subcutaneously) was administered on postoperative day (POD) 4. Two immunomodulatory therapies intended to minimize antibody rebound posttransplant, were given. Intravenous immune globulin (IVIG, Gamunex-C, Grifols, ResearchTriangle Park, NC, 2,000 mg/kg total dose) was given either as a single dose on POD7, or in two divided doses on POD7 \pm 1 , and Rituximab (Rituxan, Genentech, San Francisco, CA, 1,000 mg, intravenously) was given on POD9. Three patients had DSA rebound and antibodymediated rejection, which responded to standard of care therapies Three patients had delayed graft function, which ultimately resolved. No serious adverse events were associated with IdeS. All patients have functioning renal allografts at a median follow-up of 235 days. Three patients developed biopsy proven AMR. The earliest was observed on POD8 and the latest on POD27. Other patient developed a severe early AMR in the setting of exceptionally high-level DSA. The histopathological features of AMR were very advanced in this case, with extensive acute glomerulitis/capillaritis, inflammatory interstitial infiltrate, and interstitial hemorrhage. Following treatment with plasmapheresis, eculizumab, bortezomib, and splenic embolization, the DSA strengths decreased and the patient's renal function recovered.

IdeS as an alternative to pretransplant plasmapheresis could provide a major benefit both to patients in terms of time saved, and also to insurers as the costs of days to weeks of plasmapheresis would be exchanged for the costs of a single infusion.

Sensitized patients in need of a heart or lung transplant currently have no option but to hope for a compatible deceased donor. Next, as xenotransplantation moves toward clinical reality, one can envision a role for IdeS in eliminating antibody to nonimmunodominant epitopes that have not been eliminated by gene editing strategies. And finally, the main limitation of IdeS due to Immunogenicity, namely that it presently remains limited only to 1-2 doses, may be able to be overcome by reengineering IdeS to make it less immunostimulatory.

IdeS originates from Streptococcus pyogenes and is consequently expected to be recognized as foreign to the human immune system. The fact that all patients had detectable anti-IdeS $\operatorname{IgG}$ before dosing implies that the patients had been preexposed to IdeS, undoubtedly [64].

However, in the phase 1 study, it was demonstrated that after 6-12 months, anti-IdeS antibodies had returned to normal range. Thus, retreatment after this period may be possible and requires further investigation.

\section{Infection}

A phase 2 clinical study on the safety, immunogenicity, pharmacokinetics, and efficacy of the IgG-degrading enzyme of Streptococcus pyogenes (IdeS [imlifidase]) were assessed in a single-center, open-label ascending-dose study in highly sensitized patients with chronic kidney disease [64]. Section of Transplant Surgery at Uppsala University Hospital, were eligible for the study if they had $\geq 2$ identified HLA antibodies of which $\geq 1$ was $>3,000$ MFI in single antigen bead analysis on $\geq 2$ separate occasions. All eligible patients were pre- 
screened and tested negative for the presence of IgE antibodies to IdeS.

Eight patients with cytotoxic PRAs (median cytotoxic PRAs of 64\%) at enrollment received 1 or 2 intravenous infusions of IdeS on consecutive days $(0.12 \mathrm{mg} / \mathrm{kg}$ body weight $\times 2[n=3] ; 0.25 \mathrm{mg} /$ $\mathrm{kg} \times 1[n=3]$, or $0.25 \mathrm{mg} / \mathrm{kg} \times 2[n=2]) . \mathrm{IgG} \mathrm{deg}-$ radation was observed in all subjects after IdeS treatment, with $<1 \%$ plasma IgG remaining within $48 \mathrm{~h}$ and remaining low up to 7 days. Mean fluorescence intensity values of HLA class I and II reactivity were substantially reduced in all patients, and C1q binding to anti-HLA was abolished. IdeS also cleaved the IgG-type B cell receptor on $\mathrm{CD} 19^{+}$ memory B cells. Anti-IdeS antibodies developed 1 week after treatment, peaking at 2 weeks. A few hours after the second IdeS infusion, 1 patient received a deceased donor kidney offer. At enrollment, the patient had a positive serum crossmatch (HLA-B7), detected by complement-dependent cytotoxicity, flow cytometry, and multiplex bead assays. After IdeS infusion $(0.12 \mathrm{mg} / \mathrm{kg} \times 2)$ and when the HLA-incompatible donor (HLA-B7 ${ }^{+}$) kidney was offered, the HLA antibody profile was negative. The kidney was transplanted successfully. The percentage of both T cell and B cell PRAs was reduced in all patients within $1 \mathrm{~h}$ after IdeS treatment.

The total number of AEs reported during the study was 76, of which 27 were classified as related. There were five serious AEs (SAEs) reported. Four of these were reported as related. Three related SAEs were classified as infections and infestations: pneumonia and suspected upper respiratory infection. Myalgia was reported in two patients, $1 \mathrm{SAE}$ grade 2 . One patient experienced a suspected infusion reaction and dosing was interrupted. All symptoms were grade 1 (flushing, hypertension, hot flashes, sinus tachycardia, dyspnea, scleral hemorrhage, visual impairment) and resolved $11 \mathrm{~min}$ after the infusion was stopped. Two patients had increased levels of aspartate aminotransferase (AST) and alanine aminotransferase (ALT) reported as AEs, both of which were classified as related.

The data obtained in this phase 2 clinical study demonstrate that IdeS treatment in sensitized patients with CKD is not only effective but also safe and well tolerated and has the potential to generate a window of 7 days after treatment when HLA antibodies are reduced below the threshold level, enabling the patient to be a candidate for transplant with an organ from an HLA-incompatible donor.

\section{Complication from Anti-IL-6R mAb (Tocilizumab: TCZ)}

\section{Q: 1. What kind of side effect does the tocilizumab have? \\ 2. What is the rebound effect follow- ing tocilizumab?}

Interleukin-6 is a pleiotropic cytokine with a significant array of biologic effects on numerous cell types which include B cells, T cells, plasma cells, hepatocytes, and endothelial cells.

Interleukin-6 is not expressed in normal individuals but contributes to the clinical manifestations of inflammatory and infectious diseases. Interleukin-6 is critical for induction of T follicular cells, and B cell differentiation to plasmablast. Interleukin (IL)-6 is responsible, in conjunction with other cytokines, for normal antibody production.

IL-6 is an important mediator of inflammation that is critical to shaping $\mathrm{T}$ cell immunity and inhibiting Tregs while increasing $\mathrm{T}$ helper 17 cell (Th17) populations. IL-6 is also critical for the progression of nave $\mathrm{B}$ cells to plasmablasts and mature plasma cells. Plasmablasts also produce copious amounts of IL-6. Critical role for IL-6 produced by endothelial cells as a major factor responsible for intimal proliferation. It is accepted that IL- 6 drives CD4 T cells toward Th17 phenotype while negating Treg differentiation. Recent evidence also indicates that IL- 6 triggers IL-21 production by $\mathrm{Tfh}$, driving $\mathrm{B}$ cell maturation to plasma cells during antibody responses. Thus, IL-6 shapes T cell immunity and is a powerful stimulant for pathogenic $\operatorname{IgG}$ production and chronic antibody mediated rejection. 
Dysregulation of IL-6 production can occur in a number of disease states that ultimately are characterized by lymphadenopathy, excessive and unregulated antibody production, autoimmunity and is also associated with a deviation of $\mathrm{T}$ cell responses from Treg to Th17 with attendant inflammation and tissue injury [68].

Tocilizumab (TCZ, Actemra) is a first-in-class humanized monoclonal antibody directed at the IL-6 receptor and has been approved by FDA in the United States and other countries around the globe for the treatment of moderate to severe rheumatoid arthritis (RA) and idiopathic juvenile arthritis. Emerging data from patients with autoimmune diseases treated with TCZ suggest a potent reduction in disease targeted autoantibodies and memory B cells. In addition, recent data also suggest TCZ reduces Th 17 cells and deviates naive $\mathrm{T}$ cells to Tregs. Reports have shown that tocilizumab also reduces antibody-producing cells, diminishes inflammatory markers, and improves clinical symptomatology in a number of other autoimmune diseases. Anti-IL-6R monoclonal antibody results in significant reductions of alloantibodies, antibody production by splenic and bone marrow plasma cells, direct inhibition of plasma cell anti-HLA antibody production, and induction of $\mathrm{T}$ regulatory cells (Tregs) with inhibition of $\mathrm{T}$ follicular helper cells (Tfh).

\section{Infection}

Choi [69] identified 36 renal transplant patients with cAMR plus DSAs and TG who failed standard of care treatment with IVIg plus rituximab with or without plasma exchange. Patients were offered rescue therapy with the anti-IL-6 receptor monoclonal antibody (tocilizumab) with monthly infusions and monitored for DSAs and long-term outcomes. Most patients showed progressive renal dysfunction and had failed treatment with IVIg plus rituximab with or without plasma exchange.

Between 2011 and 2016, 36 patients were offered treatment with tocilizumab $(8 \mathrm{mg} / \mathrm{kg}$ monthly, maximal dose $800 \mathrm{mg}$ for 6-25 months), based on insurance approval.

The median follow-up was 3.26 years (IQR $1.82-3.81$ ) with a maximal follow-up of 8 years.

The patients treated with tocilizumab exhibited good allograft survival with a graft survival probability of $80 \%$ at 6 years post-cAMR diagnosis. Tocilizumab-treated patients with TG exhibited good allograft survival with a graft survival probability of $77 \%$ at 6 years post cAMR. The patient survival was good at 6 years with a survival probability of $91 \%$.

However, eGFRs for both cohorts remained stable during the study period. Regarding the impact of tocilizumab on Immunodominant (iDSA) levels, which are defined as the strongest DSAs detected in the patients' sera, declined significantly beginning at 24 months.

Among the 36 recipients treated with tocilizumab, only four had a graft loss (11.1\%), which were due to cAMR.

Of interest, tocilizumab was discontinued for medical reasons in one patient and for financial reasons in the other three about 6 months before all graft losses were seen. Although it is uncertain, we must consider the possibility that rebound in IL-6-IL-6R signaling after cessation of tocilizumab is responsible for the initiation of alloimmune response and allograft loss.

Thirteen patients had infectious AEs: a total of five patients had cytomegalovirus infection, two patients had polyoma BK infection that resolved with treatment, and one patient was diagnosed with trichodysplasia spinulosa (a benign skin condition related to polyoma virus) that resolved 1 month after the completion of tocilizumab therapy. Seven patients had bacterial infections, which resolved with treatment, usually without the need for cessation of tocilizumab therapy. All infectious events resolved with directed treatment and without the need to stop tocilizumab therapy. One patient had transient visual disturbance with resolution. Eight patients developed hypogammaglobulinemia defined by $\operatorname{IgG}<600 \mathrm{mg} /$ $\mathrm{dL}$ during tocilizumab therapy.

No significant adverse events or severe adverse events were seen. Tocilizumab provides good long-term outcomes for patients with cAMR and 
TG, especially compared with historical published treatments.

Patel [70] reported that since 2015, 20 pts. received TCZ $8 \mathrm{mg} / \mathrm{kg}$ with $>3$ months follow-up added to tacrolimus/mycophenolate/pred for cAAMR refractory to treatment. Mean age at KTx was $37 \pm 11.5$ years, most were female [13], and received live donor KTx [11]. All patients had prior AMR with DSA-Class I = 4, Class II = 9, Class I and $\mathrm{II}=7$ - that persisted despite plasmapheresis [6], IVIG at $2 \mathrm{~g} / \mathrm{kg}$ [8], rituximab [2]. Sixteen patients also had prior ACR (borderline $=9$, $1 \mathrm{~A}=3,1 \mathrm{~B}=3,2 \mathrm{~A}=1$ ). TCZ was started an average 1,648 $\pm 1,420$ days after transplant, with a starting creatinine- $=2.35 \pm 0.95$, and given an average $323 \pm 281$ days. In the 3 months prior to initiation of TCZ, eGFR declined by $3.9 \mathrm{cc} / \mathrm{min}$ each month, compared to $0.05 \mathrm{cc} / \mathrm{min}$ each month on TCZ $(p=0.008)$. Proteinuria also stabilized on TCZ- initial urine protein:creatinine ratio of $1.01( \pm 1.1)$ vs. $0.80( \pm 1.1)$ at $\mathrm{f} / \mathrm{u}$. Stabilization was not dependent on level or type of DSA, and nor did DSA change significantly during follow-up. There were 3 ACRs (2-borderline and $1 \mathrm{~B}=1$ ) and one patient with recurrent AMR after stopping TCZ that responded to re-initiation. There were 2 cases of BK viremia (0 nephropathy), $1 \mathrm{EBV}$ viremia, and 1 hospitalization for pneumonia. Only one patient stopped due to infusion-related reaction.

\section{Others}

Vo et al. [71, 72] reported on the efficacy of tocilizumab in reducing anti-HLA antibodies and improving transplant rates in highly HLAsensitized patients who were resistant to other desensitization strategies. This, phase I/II single center open label pilot exploratory study was conducted at Cedars-Sinai Medical Center, in which from July 2012 to November 2013, ten patients unresponsive to desensitization (DES) with IVIg + rituximab were treated with IVIg + TCZ. Patients received IVIg on days 0 and 30 at $2 \mathrm{~g} / \mathrm{kg}$ and TCZ $8 \mathrm{mg} / \mathrm{kg}$ on day 15 then monthly for 6 months. If transplanted, patients received IVIg once and TCZ monthly for
6 months. Patients received alemtuzumab $30 \mathrm{mg}$ subcutaneously as induction and were maintained on triple regimen with tacrolimus MMF and prednisone taper.

Five of 10 patients were transplanted. Mean time to transplant from first DES was $25 \pm 10.5$ months but after TCZ was $8.1 \pm 5.4$ months. Six-month protocol biopsies showed no antibody-mediated rejection. However, 1 patient showed mild ABMR on 12 months for cause biopsy, 6 months after completion of the TCZ dosing. This patient responded well to ABMR treatment with IVIg + rituximab. Donor-specific antibody strength and number were reduced by TCZ treatment. Renal function at 12 months was $60 \pm 25 \mathrm{~mL} / \mathrm{min}$.

Tocilizumab and IVIg appear to be safe. From this pilot trial, they are cautiously optimistic that targeting the IL-6/IL-6R pathway could offer a novel alternative for difficult to desensitize patients. The adverse effects included nausea, abdominal pain with normal amylase and lipase, itching, fatigue, blurred vision, anemia, thrombocytopenia, liver function test abnormalities 1.5 or less normal, elevated blood pressures, and minimal infusion-related reactions to IVIg + TCZ.

In the transplanted group: 1 patient developed infective colitis with colonic perforation required bowel resection (possibly related to study drug) and 1 patient developed Bell Palsy (possibly related to study drug). The clinical correlates include an association with amyloid A amyloidosis, increased risk for development of cardiovascular complications, and the anemia of chronic disease associated with hepcidin elevation.

They also saw significant reductions in $\mathrm{T}$ follicular cell populations and increases in Treg cells after anti-IL-6R therapy. Enhanced Treg responses have also been reported in humans treated with TCZ. They tested the hypothesis that IL-6 is an important cytokine in the maintenance of anti-HLA antibody production in highly sensitized patients.

Choi et al. [73] report on their extended experience with $\mathrm{TCZ}$ for CABMR+TG. Methods: Since 4/2011 they identified 65 patients including those with CABMR+TG, DSA+, and/or 
AT1R ab+. TCZ treatment was pursued after other treatments had failed. Briefly, after diagnosis of CABMR, patients received TCZ $4-8 \mathrm{mg} / \mathrm{kg}$ monthly for 3-37 doses and were followed up to 6 years from TCZ initiation.

Immunodominant (iDSA) levels tended to decrease after therapy ( $\mathrm{t} 0: 12,967 \pm 20,000$, t12 M: 9,180 $\pm 6,682, \mathrm{t} 36 \mathrm{M}: 3,829 \pm 6,001 \mathrm{MFI})$ $(p=\mathrm{NS})$. Mean eGFRs were $53.18 \pm 34.61 \mathrm{~mL} /$ $\min$ at $0 \mathrm{M}$ vs. $50.43 \pm 36.37 \mathrm{~mL} / \mathrm{min}$ at $24 \mathrm{M}$. Graft survival was compared to a standard (SOC) group (39-non concurrent CABMR patients) treated with IVIg + rituximab \pm PLEX. At 6 years, $92.6 \%$ of TCZ patients have functioning grafts $v .53 .3 \%$ in SOC $(p=0.0005)$. Two deaths in the TCZ group. Preand post-TCZ biopsies at mean of $29.5 \pm 18.7 \mathrm{M}$ from pre-biopsy showed significant reductions in $\mathrm{g}+$ ptc scores compared to biopsy at diagnosis.

\section{Complication from Plasmapheresis}

\section{Q: 1. What kind of side effect does plas- mapheresis have?}

2. Are the complication from plasmapheresis preventable?

Szczeklik [74] analyzed adverse effects of 370 plasmapheresis procedures in 54 patients in intensive care unit with disease included myasthenia gravis $(33.3 \%)$, Guillain-Barre syndrome, Lyell's syndrome (9.3\%), SLE (7.4\%), and PTT (7.4\%).

The adverse side effects observed most frequently during plasma filtration were: fall in arterial blood pressure, arrhythmias, sensations of cold with temporarily elevated temperature, and paresthesias.

Severe and life-threatening episodes, i.e., shock, fall in arterial blood pressure requiring pressor amines, persistent arrhythmias and hemolysis, developed in $2.16 \%$ of procedures.

The adverse-side effects are associated with large vessel catheterization, clotting disorders, septic complications resulting from impaired immunity caused by the removal of antibodies during the procedure, catheter-associated infections, and those related to transfusion of blood products. Moreover, life-threatening fall in arterial blood pressure, cardiac arrhythmias and water-electrolyte imbalance are likely to develop. Less severe reactions and symptoms are more common, e.g., urticaria, pruritus, limb paresthesias and pains, muscle contractions, dizziness, nausea, vomiting, transiently elevated temperature, shivers, seizures, head and chest pains. Reduced levels of hemoglobin, thrombocytopenia, hypokalemia, and reduced concentrations of fibrinogen were developed. The total incidence of complications is estimated at $25-40 \%$. Lifethreatening and non-life-threatening complications were developed.

Zhang et al. [75] analyzed the 28 enrolled patients diagnosed serologically and pathologically with anti-GBM disease from 2003 to 2013 in whom 16 treated with DFPP and 12 with IA,

A double volume of plasma was processed, and each patient received a 30-40 g human albumin supplement during each session. IA consisted of 10 cycles per session, with 8-10 sessions performed daily or every other day and each session regenerating 30-60 L of plasma.

Six patients had pulmonary hemorrhage and 18 had serum creatinine concentrations $>500$ umol/L. All patients showed progressive increases in serum creatinine and required CRRT during the course of disease. Efficacy of clearing anti-GBM antibody was similar in the two groups. One patient each had a pulmonary hemorrhage and a subcutaneous hemorrhage during treatment, but there were no other serious complications. At the end of follow-up, patient survival and renal survival were similar in the DFPP and IA groups. DFPP plus immunosuppressive therapy efficiently and safely removed anti-GBM antibodies. The fewer plasma-associated side effects and reduced loss of IgG suggest that DFPP may be a better treatment choice for anti-GBM disease, especially in patients with insufficient plasma. 


\section{Surgical Complication}

Q: 1. What kind of surgical complications develop following desensitization?

2. What are the strategies for their prevention?

\section{Lymphocele}

Zschiedrich et al. [76] analyzed 100 ABOi KTx and 248 ABOc KTx in observational, single center study. Preoperative desensitization were single dose of rituximab $\left(375 \mathrm{mg} / \mathrm{m}^{2}\right) 30$ days before operation, and immunoadsorption. Seven days before surgery, oral immunosuppression with tacrolimus and MMF and prednisone was initiated followed by basiliximab induction.

In this study postoperative lymphoceles occurred more frequently in ABOi KTx.

\section{Bleeding}

A single-center retrospective study by Naciri et al. [18] assessed posttransplantation complications in $44 \mathrm{ABO}-\mathrm{i}$ versus 44 matched ABO-c patients. All patients were comparable at baseline except that ABO-i patients had greater immunological risks.

Preoperative desensitization were single dose of rituximab $\left(375 \mathrm{mg} / \mathrm{m}^{2}\right) 30$ days before operation, and immunoadsorption. Twelve days before surgery, oral immunosuppression with tacrolimus and MMF and prednisone was initiated followed by basiliximab induction.

During the 6-month posttransplant period, more $\mathrm{ABO}-\mathrm{i}$ patients presented with postoperative bleeds, thus requiring significantly more blood transfusions.

Habicht [16] retrospectively analyzed 21 consecutive recipients who underwent $\mathrm{ABO}$ i renal transplantation. Pretransplant desensitization included administration of rituximab $(375 \mathrm{mg} /$ $\mathrm{m}^{2}$ ), mycophenolate mofetil (MMF), tacrolimus and prednisolone 4 weeks prior of scheduled transplantation as well as IA and IVIG.
Hemorrhagic complications occurred in $9.5 \%$ of the $\mathrm{ABO}$ recipients as opposed to $2 \%$ in the $\mathrm{ABOc}$ group. The risk of lymphoceles requiring drainage or surgical repair and major wound-healing problems was increased in the ABOi group as compared to the ABOc group (19\% vs. 6.4\%). Proximal ureteral stenosis developed and required surgical repair 2 months after transplantation. Extensive histologic and immunohistochemical workup revealed CMV uretritis as the cause of stenosis. Interestingly, the most common viral infection was BK viremia leading to $\mathrm{BKN}$ in 5 of $20 \mathrm{ABOi}$ grafts $(25 \%)$ and 4 of $47(8.5 \%)$ ABOc grafts.

Szezeklik [74] reported that during the 6-month posttransplant period, more ABO-i patients presented with postoperative bleeds, thus requiring significantly more blood transfusions.

Patient- and graft-survival rates, and kidneyfunction statuses were similar between both groups at 6 months posttransplantation.

It is reported that ABOi patients have more bleeding episodes either during transplantation or in the immediate posttransplant period [5-9]. This has been largely ascribed to apheresis session(s).

Significantly more lymphoceles (which require specific therapies) as well as significantly more wound dehiscences have been reported in ABOi patients.

Lentine [17] reported that recipients of ABOi transplants experienced significantly $(P<0.05)$ higher incidence of wound infections $(12.7 \%$ vs. $7.3 \%$ ), pneumonia ( $7.6 \%$ vs. $3.8 \%$ ), and urinary tract infections (UTIs) or pyelonephritis $(24.5 \%$ vs. $15.3 \%$ ) in the first 90 days compared with ABO-compatible recipients. In adjusted models, $\mathrm{ABO}$ incompatibility was associated with twice the risk of pneumonia (adjusted hazard ratio [aHR], 2.22; 95\% confidence interval [CI], 1.144.33 ) and $56 \%$ higher risk of UTIs or pyelonephritis (aHR 1.56; 95\% CI 1.05-2.30) in the first 90 posttransplantation days, and 3.5 times the relative risk of wound infections in days 91 to 365 (aHR 3.55; 95\% CI 1.92-6.57). ABOi recipients, $19 \%$ of whom underwent pre- or peritransplant splenectomy, experienced twice the adjusted risk of early hemorrhage.

ABOi transplantation offers patients with potential live donors an additional transplant 
option but with higher risks of infectious and hemorrhagic complications. Awareness of these complications may help improve protocols for the management of ABOi transplantation.

In our center (Asan Medical Center) ABOi KT was performed on 276 patients (2009-2015) and a total 96 patients underwent FCXM positive KT. The overall patient survival rate in patients who underwent FXCM postivie KT was not significantly different from that of the control group during the 3-year follow-up $(P=0.34)$.

After desensitization with plasmapheresis and rituximab the FXCM positive group showed a higher rate of surgical complications-including hematoma (3.7\% vs. $20.0 \%, P<0.001)$, bleeding requiring operation $(1.0 \%$ vs. $6.7 \%, P=0.002)$, and lymphocele $(2.7 \%$ vs. $8.9 \%, P=0.020)-$ than the FXCM negative group. Infectious complications, however, demonstrated no significant differences [66].

\section{References}

1. Keith DS, Vranic GM. Approach to the highly sensitized kidney transplant candidate. Clin J Am Soc Nephrol. 2016 Apr 7;11(4):684-93. https://doi. org/10.2215/CJN.05930615.

2. Tsutsumi Y, Yamamoto Y, Shimono J, et al. Hepatitis $\mathrm{B}$ virus reactivation with rituximab-containing regimen. World J Hepatol. 2013;5(11):612-20.

3. Chung BH, Yun JT, Ha SE, Kim JI, Moon IS, Choi BS, Park CW, Kim YS, Yang CW. Combined use of rituximab and plasmapheresis pre-transplant increases post-transplant infections in renal transplant recipients with basiliximab induction therapy. Transpl Infect Dis. 2013;15(6):559-68.

4. Nakao T, Ushigome H, Kawai K, et al. Evaluation of rituximab dosage for $\mathrm{ABO}$-incompatible livingdonor kidney transplantation. Transplant Proc. 2015;47(3):644-8.

5. Yang SH, Kuo SH. Reactivation of hepatits B virus during rituximab treatment of a patient with follicular lymphoma. Ann Hematol. 2009;87:325-7.

6. Huang YH, Hsiao LT, Hong YC, et al. Randomized controlled trial of entecavir prophylaxis for rituximab-associated hepatitis $B$ virus reactivation in patients with lymphoma and resolved hepatitis B. J Clin Oncol. 2013;31(22):2765-72.

7. Loomba R, Rowley A, Wesley R, et al. Systematic review: the effect of preventive lamivudine on hepatitis $\mathrm{B}$ reactivation during chemotherapy. Ann Intern Med. 2008;148(7):519-28.
8. Mozessohn L, Chan KK, Feld JJ, Hicks LK. Hepatitis $\mathrm{B}$ reactivation in $\mathrm{HBs}$ g-negative/ $\mathrm{HBcAb}$-positive patients receiving rituximab for lymphoma: a metaanalysis. J Viral Hepat. 2015;22(10):842-9.

9. Lee J, Park JY, Huh KH, Kim BS, et al. Rituximab and hepatitis B reactivation in HBsAg-negative/anti$\mathrm{HBc}$-positive kidney transplant recipients. Nephrol Dial Transplant. 2017;32(4):722-9.

10. Tallantyre EC, Whittam DH, Jolles S, et al. Correction to: secondary antibody deficiency: a complication of anti-CD20 therapy for neuroinflammation. J Neurol. 2018;265(5):1123.

11. Healy EG, Phadke R, Kidd M, Reilly MM, Lunn MP. Clinical, neuropathological and radiological evidence for a rare complication of rituximab therapy. Neuromuscul Disord. 2015;25(7):589-92.

12. Shah S, Jaggi K, Greenberg K, Geetha D. Immunoglobulin levels and infection risk with rituximab induction for anti-neutrophil cytoplasmic antibody-associated vasculitis. Clin Kidney J. 2017;10(4):470-4.

13. Okada M, Watarai Y, Iwasaki K, et al. Favorable results in $\mathrm{ABO}$-incompatible renal transplantation without B cell-targeted therapy: advantages and disadvantages of rituximab pretreatment. Clin Transpl. 2017;31(10):e13071.

14. Morath C, Zeier M, Döhler B, Opelz G, Süsal C. ABO-Incompatible kidney transplantation. Front Immunol. 2017;8:234.

15. Morath C, Zeier M, Süsal C. Increased risk of infection-associated death with incompatible kidney transplantations. Transpl Int. 2017;30(12):1209-11.

16. Habicht A, Bröker V, Blume $C$, et al. Increase of infectious complications in ABO-incompatible kidney transplant recipients - a single centre experience. Nephrol Dial Transplant. 2011;26(12):4124-31.

17. Lentine KL, Axelrod D, Klein C, et al. Early clinical complications after ABO-incompatible live-donor kidney transplantation: a national study of medicareinsured recipients. Transplantation. 2014;98(1):54-65.

18. Naciri Bennani H, Abdulrahman Z, Allal A, et al. Early post-transplant complications following ABOincompatible kidney transplantation. J Nephropathol. 2016;5(1):19-27.

19. Opelz G, Morath C, Süsal C, et al. Three-year outcomes following 1420 ABO-incompatible livingdonor kidney transplants performed after $\mathrm{ABO}$ antibody reduction: results from 101 centers. Transplantation. 2015;99(2):400-4.

20. Barnett AN, Manook M, Nagendran M, et al. Tailored desensitization strategies in $\mathrm{ABO}$ blood group antibody incompatible renal transplantation. Transpl Int. 2014;27(2):187-96.

21. Choi BH, Cho HK, Jung JH, et al. How to reduce lethal infectious complications in ABO-incompatible kidney transplantation. Transplant Proc. 2015;47(3):653-9.

22. Choi BH, Han DJ. Ongoing higher infection rate in ABO-incompatible kidney transplant recipient: is it a serious problem? A single-center experience. Ann Surg Treat Res. 2016;91:37. 
23. Kwon H, Kim YH, Choi JY, et al. Analysis of 4000 kidney transplantations in a single center: across immunological barriers. Medicine (Baltimore). 2016;95(32):e4249.

24. Trivin C, Tran A, Moulin B, et al. Infectious complications of a rituximab-based immunosuppressive regimen in patients with glomerular disease. Clin Kidney J. 2017;10(4):461-9.

25. Schrezenmeier E, Budde K, Staeck O, et al. Incidence of infectious disease and malignancies after rituximab therapy in kidney transplant recipients: results from a cohort in germany. Transplant Proc. 2017;49(10):2269-73.

26. Clatworthy MR, Watson CJ, Plotnek G, et al. B-celldepleting induction therapy and acute cellular rejection. N Engl J Med. 2009;360(25):2683-5.

27. Agarwal A, Vieira CA, Book BK, Sidner RA, Fineberg NS, Pescovitz MD. Rituximab, anti-CD20, induces in vivo cytokine release but does not impair ex vivo T-cell responses. Am J Transplant. 2004 Aug;4(8):1357-60.

28. Lioté H, Lioté F, Séroussi B, et al. Rituximab-induced lung disease: a systematic literature review. Eur Respir J. 2010;35(3):681-7.

29. Wan Zaidi WA, Wan Jamaludin WF, Tumian NR, Abdul Wahid SF. Rituximab-induced lung disease. Med J Malaysia. 2016;71(4):209-10.

30. Hadjinicolaou AV, Nisar MK, Parfrey H, et al. Noninfectious pulmonary toxicity of rituximab: a systematic review. Rheumatology (Oxford). 2012;51(4):653-62.

31. Child N, O'Carroll M, Berkahn L. Rituximabinduced interstitial lung disease in a patient with immune thrombocytopenia purpura. Intern Med J. 2012;42(3):e12-4.

32. Ahn SH, Kim SM, Sung JJ. Rituximab-induced interstitial lung disease in a patient with aquaporin-4 immunoglobulin G-positive neuromyelitis optica spectrum disorder. Mult Scler Relat Disord. 2018;20:192-3.

33. Verma SK. Updated cardiac concerns with rituximab use: a growing challenge. Indian Heart J. 2016;68(Suppl 2):S246-8.

34. Ureshino H, Nishioka A, Kojima $K$, et al. Rituximab-induced acute thrombocytopenia in high tumor burden follicular lymphoma. Intern Med. 2016;55(15):2061-4.

35. Blanco B, Pérez-Simón JA, Sánchez-Abarca L, et al. Treatment with bortezomib of human CD4+ T cells preserves natural regulatory $\mathrm{T}$ cells and allows the emergence of a distinct suppressor T-cell population. Haematologica. 2009;94(7):975-83.

36. Jeong JC, Jambaldorj E, Kwon HY, Kim MG, Im HJ, Jeon HJ, In JW, Han M, Koo TY, Chung J, Song EY, Ahn C, Yang J. Desensitization using bortezomib and high-dose immunoglobulin increases rate of deceased donor kidney transplantation. Medicine (Baltimore). 2016;95(5):e2635.

37. Shah N, Meouchy J, Qazi Y. Bortezomib in kidney transplantation. Curr Opin Organ Transplant. 2015;20(6):652-6. MOT.0000000000000252.
38. Moreno Gonzales MA, Gandhi MJ, Schinstock CA, et al. Doses of bortezomib for desensitization is not well tolerated and is associated with only modest reductions in anti-HLA antibody. Transplantation. 2017;101(6):1222-7.

39. Eskandary F, Regele H, Bond G, et al. Bortezomib in late antibody-mediated kidney transplant rejection-a double-blind randomized placebo-controlled trial (BORTEJECT study). Am J Transplant. 2018;18(S4):326.

40. Eskandary F, Regele H, Baumann L, et al. A randomized trial of bortezomib in late antibody-mediated kidney transplant rejection. J Am Soc Nephrol. 2018;29(2):591-605.

41. Kwun J, Burghuber C, Manook M, et al. Humoral compensation after bortezomib treatment of allosensitized recipients. J Am Soc Nephrol. 2017;28(7):1991-6.

42. Lemy A, Toungouz M, Abramowicz D. Bortezomib: a new player in pre- and post-transplant desensitization? Nephrol Dial Transplant. 2010;25(11):3480-9.

43. Rault R, Piraino B, Johnston JR, Oral A. Pulmonary and renal toxicity of intravenous immunoglobulin. Clin Nephrol. 1991;36(2):83-6.

44. Pierce LR, Jain N. Risks associated with the use of intravenous immunoglobulin. Transfus Med Rev. 2003;17(4):241-51.

45. Sin YH, Kim YJ, Oh JS, et al. Graft rupture after high-dose intravenous immunoglobulin therapy in a renal transplant patient. Nephrology (Carlton). 2014;19(Suppl 3):35-6.

46. Vo AA, Cam V, Toyoda M, et al. Safety and adverse events profiles of intravenous gammaglobulin products used for immunomodulation: a single-center experience. Clin J Am Soc Nephrol. 2006;1(4):844-52.

47. Jordan S, Cunningham-Rundles C, McEwan R. Utility of intravenous immune globulin in kidney transplantation: efficacy, safety, and cost implications. Am J Transplant. 2003;3(6):653-64.

48. Kahwaji J, Barker E, Pepkowitz S, et al. Acute hemolysis after high-dose intravenous immunoglobulin therapy in highly HLA sensitized patients. Clin J Am Soc Nephrol. 2009;4(12):1993-7.

49. Welsh KJ, Bai Y. Therapeutic plasma exchange as a therapeutic modality for the treatment of IVIG complications. J Clin Apher. 2015;30(6):371-4.

50. Stenton SB, Dalen D, Wilbur K. Myocardial infarction associated with intravenous immune globulin. Ann Pharmacother. 2005;39(12):2114-8.

51. Kakuta Y, Satoh S, Watarai Y, et al. Transplant recipients using plasmapheresis and super high-dose intravenous immunoglobulin. Transplant Direct. 2017;4(1):e336.

52. Davis J. Eculizumab. Am J Health Syst Pharm. 2008;65(17):1609-15.

53. Cornell LD, Schinstock CA, Gandhi MJ, et al. Positive crossmatch kidney transplant recipients treated with eculizumab: outcomes beyond 1 year. Am J Transplant. 2015;15(5):1293-302.

54. Stegall MD, Diwan T, Raghavaiah S, et al. Terminal complement inhibition decreases antibody-mediated 
rejection in sensitized renal transplant recipients. Am J Transplant. 2011;11(11):2405-13.

55. West-Thielke P, Progar K, Campara M, et al. Eculizumab for prevention of antibody-mediated rejection in blood group-incompatible renal transplantation. Transplant Proc. 2018;50(1):66-9.

56. Johnson CK, Leca N. Eculizumab use in kidney transplantation. Curr Opin Organ Transplant. 2015;20(6):643-51.

57. Kulkarni S, Kirkiles-Smith NC, Deng YH, et al. Eculizumab therapy for chronic antibody-mediated injury in kidney transplant recipients: a pilot randomized Controlled trial. Am J Transplant. 2017;17(3):682-91.

58. Tillou X, Poirier N, Le Bas-Bernardet S, et al. Recombinant human $\mathrm{C} 1$-inhibitor prevents acute antibody-mediated rejection in alloimmunized baboons. Kidney Int. 2010;78(2):152-9.

59. Jordan SC, Choi J, Aubert O, et al. A phase I/II, double-blind, placebo-controlled study assessing safety and efficacy of $\mathrm{C} 1$ esterase inhibitor for prevention of delayed graft function in deceased donor kidney transplant recipients. Am J Transplant. 2018;18:2955.

60. Vo AA, Zeevi A, Choi J, et al. A phase I/II placebocontrolled trial of $\mathrm{C} 1$-inhibitor for prevention of antibody-mediated rejection in HLA sensitized patients. Transplantation. 2015;99(2):299-308.

61. Montgomery RA, Orandi BJ, Racusen L, et al. Plasma-derived $\mathrm{C} 1$ esterase inhibitor for acute antibody-mediated rejection following kidney transplantation: results of a randomized double-blind placebo-controlled pilot study. Am J Transplant. 2016;16(12):3468-78.

62. Viglietti D, Gosset C, Loupy A, et al. C1 Inhibitor in acute antibody-mediated rejection nonresponsive to conventional therapy in kidney transplant recipients: a pilot study. Am J Transplant. 2016;16(5):1596-603.

63. Järnum S, Bockermann R, Runström A, et al. The bacterial enzyme IdeS cleaves the IgG-type of B cell receptor (BCR), abolishes BCR-mediated cell signaling, and inhibits memory B cell activation. J Immunol. 2015;195(12):5592-601.

64. Lorant T, Bengtsson M, Eich T, et al. Safety, immunogenicity, pharmacokinetics, and efficacy of degradation of anti-HLA antibodies by IdeS (imlifidase) in chronic kidney disease patients. Am J Transplant. 2018;18:2752-62.

65. Jordan SC, Lorant T, Choi J, et al. IgG endopeptidase in highly sensitized patients undergoing transplantation. N Engl J Med. 2017;377:442-53.
66. Huang E, Jordan SC. Immunoglobulin G-degrading enzyme of Streptococcus pyogenes (IdeS), desensitization, and the kidney allocation system: complementary approaches to Increase transplantation in highly HLA sensitized patients. Clin J Am Soc Nephrol. 2018;13(5):799-801.

67. Lonze BE, Tapapudi VS, et al. IdeS (imlifi dase): a novel agent that cleaves human IgG and permits successful kidney transplantation across high-strength donor-specific antibody. Ann Surg. 2018;268(3):488-96.

68. Kishimoto T. IL-6: from its discovery to clinical applications. Int Immunol. 2010;22(5):347-52.

69. Choi J, Aubert O, Vo A, et al. Assessment of tocilizumab (anti-interleukin-6 receptor monoclonal) as a potential treatment for chronic antibody-mediated rejection and transplant glomerulopathy in HLAsensitized renal allograft recipients. Am J Transplant. 2017;17(9):2381-9.

70. Pate S, Mohan S, Fernandez H, et al. Tocilizumab stabilizes renal function in kidney transplant recipients with chronic active antibody mediated rejection (CAAMR). Am J Transplant. 2018;18(S4):326.

71. Vo AA, Choi J, Kim I, et al. A phase I/II trial of the interleukin-6 receptor-specific humanized monoclonal (tocilizumab) + intravenous immunoglobulin in difficult to desensitize patients. Transplantation. 2015;99(11):2356-63.

72. Jordan SC, Choi J, Kim I, et al. Interleukin-6, a cytokine critical to mediation of inflammation, autoimmunity and allograft rejection: therapeutic implications of IL-6 receptor blockade. Transplantation. 2017;101(1):32-44.

73. Choi J, Aubert O, Louie S, et al. Extended experience using tocilizumab (anti-IL6R, TCZ) for the treatment of chronic antibody mediated rejection (CABMR). Am J Transplant. 2018;18(S4):326.

74. Szczeklik W, Wawrzycka K, Włudarczyk A, Sega A, Nowak I, Seczyńska B, Fajfer I, Zając K, Królikowski W, Kózka M. Complications in patients treated with plasmapheresis in the intensive care unit. Anaesthesiol Intensive Ther. 2013;45(1):7-13.

75. Zhang YY, Tang Z, Chen DM, et al. Comparison of double filtration plasmapheresis with immunoadsorption therapy in patients with anti-glomerular basement membrane nephritis. BMC Nephrol. 2014;15:128.

76. Zschiedrich S, Jänigen B, Dimova D, et al. One hundred $\mathrm{ABO}$-incompatible kidney transplantations between 2004 and 2014: a single-centre experience. Nephrol Dial Transplant. 2016;31(4):663-71. 